\title{
Regulation of adult neurogenesis and neuronal differentiation by Neural Cell Adhesion Molecule 2 (NCAM2)
}

\author{
1 Alba Ortega-Gascó ${ }^{1,2,+}$, Antoni Parcerisas ${ }^{1,2,3,+}$, Keiko Hino $^{4}$, Vicente Herranz-Pérez ${ }^{2,5,6}$, \\ 2 Fausto Ulloa ${ }^{1,2}$, Alba Elias-Tersa ${ }^{1,2}$, Miquel Bosch ${ }^{3}$, José Manuel García-Verdugo ${ }^{2,5}$, \\ 3 Sergi Simó ${ }^{4}$, Lluís Pujadas ${ }^{1,2, \#, *}$ and Eduardo Soriano ${ }^{1,2, *}$. \\ $4{ }^{1}$ Department of Cell Biology, Physiology, and Immunology; Institute of Neurosciences; \\ 5 University of Barcelona, Barcelona, Spain. \\ $6 \quad{ }^{2}$ Centro de Investigación Biomédica en Red Sobre Enfermedades Neurodegenerativas \\ 7 (CIBERNED), Madrid, Spain. \\ $8{ }^{3}$ Department of Basic Sciences, Universitat Internacional de Catalunya Barcelona, Sant \\ 9 Cugat del Vallès, Spain. \\ $10{ }^{4}$ Department of Cell Biology and Human Anatomy, University of California, Davis, \\ 11 California, USA.
}

$12{ }^{5}$ Laboratory of Comparative Neurobiology, Cavanilles Institute of Biodiversity and 13 Evolutionary Biology, University of Valencia, Spain.

$14{ }^{6}$ Predepartamental Unit of Medicine, Faculty of Health Sciences, Universitat Jaume I, 15 Castelló de la Plana, Spain.

16 "Present address: Faculty of Heath Sciences and Wellfare; Tissue Repair and 17 Regeneration Laboratory (TR2Lab); University of Vic - Central University of Catalonia 18 (UVic-UCC), Vic, Catalonia, Spain

$19+$ AO-G and AP contributed equally.

* Correspondence: Professor Eduardo Soriano; esoriano@ub.edu, Lluís Pujadas; 21 Iluis.pujadas@ub.edu.

22 Keywords: cell adhesion molecules, adult neurogenesis, radial glial progenitor cells, 23 corticogenesis, neuronal migration. 


\section{$24 \quad$ ABSTRACT}

25 Adult neurogenesis persists in mammals in the neurogenic zones where newborn 26 neurons are incorporated into existing neuronal circuits. Relevant molecular elements 27 of the neurogenic niches include the family of Cell Adhesion Molecules (CAM), which participate in signal transduction and regulate radial glial progenitor's (RGPs) survival, division and differentiation. The Neural Cell Adhesion Molecule 2 (NCAM2) is expressed

30 in brain development and in adult stages, and controls dendrite arborisation and 31 synaptic formation and maintenance during development. Nevertheless, the role of 32 NCAM2 in neurogenesis and lineage progression is not well understood. Here we 33 analyse the functions of NCAM2 in the regulation of RGPs in adult neurogenesis in the dentate gyrus and during corticogenesis, by using different lentiviral-mediated genetic approaches to modulate its expression, both in vivo and in vitro. First, we characterized the expression of NCAM2 among the main actors of the neurogenic process revealing different levels of NCAM2 amid the progression of RGPs and the formation of juvenile neurons. Further, we show that overexpression of NCAM2 arrest infected cells in a RGP-

39 like state, with characteristic morphological, immunocytochemical and electron

40 microscopy features. In contrast, NCAM2 overexpression in embryonic cortical 41 progenitors does not seems to alter cell fate, but causes transient migration deficits. 42 These results reveal a differential role of NCAM2 in the regulation of adult and embryonic RGPs, and specifically, a significant implication of NCAM2 in the regulation and progression of RGPs during adult neurogenesis in the hippocampus. 
In mammals, active neurogenesis is preserved during adulthood in specific niches (Altman and Das 1965) by remaining radial glia progenitors (RGPs) in the subventricular zone (SVZ) of the lateral ventricles and in the subgranular zone (SGZ) of the hippocampal dentate gyrus (DG) (Gonçalves et al. 2016; Gage 2019; Ghosh 2019; Kumar et al. 2019; Denoth-Lippuner and Jessberger 2021). Adult neurogenesis recapitulates the developmental processes including proliferation, neuronal fate specification, migration, differentiation, synaptogenesis, and functional integration into preexistent circuits. It has been shown that neurogenesis in the adult brain plays an important role in memory and learning processes (Zhao et al. 2008; Bergmann et al. 2015; Kumar et al. 2019).

RGPs are located in specialized microenvironments or neurogenic niches where they are subjected to multiple signaling pathways that control its maintenance, proliferation and lineage progression. Those extrinsic and intrinsic cues include cytokines, trophic and growth factors, neurotransmitters, epigenetic mechanisms as well as physiological and pathological variables (Yao et al., 2016; Zhang \& Sheng et al., 2015; Zhang, 2018; Zhao et al., 2008). Cell adhesion molecules (CAMs) have also been revealed as essential components of these microenvironments. They not only sustain the cytoarquitechture of the niche but also provides a link between the extracellular and the intracellular domains of RGPs participating in signal transduction. Therefore, CAMs are important for self-renewal and proliferation of RGPs, and for neuronal differentiation and migration (Bian 2013; Morante-Redolat and Porlan 2019).

The mammalian neural cell adhesion molecule (NCAM) family is composed of two members, NCAM1 and NCAM2, sharing a similar structure of 5 immunoglobulin domains and 2 fibronectin type III domains, but presenting different expression patterns, post-transcriptional modifications, and molecular interactions (Pébusque et al. 1998; Makino and McLysaght 2010; Parcerisas, Ortega-gascó, et al. 2021). NCAM1 has been extensively studied and it has been described to play a role in neuronal migration, neurite development, synaptogenesis, and also in neurogenesis by regulating embryonic and adult neural stem cells (NSCs) (Kiselyov et al. 2003; Bonfanti 2006; Angata et al. 
75 NCAM2.1, with a cytoplasmatic domain, and NCAM2.2, which is GPI-anchored (Von 76 Campenhausen et al. 1997; Alenius and Bohm 2003). In the central nervous system 77 (CNS), the functions of NCAM2 have been mainly linked to the regulation of the 78 formation and maintenance of axonal and dendritic biology compartments in the olfactory system (Alenius \& Bohm, 2003; Kulahin \& Walmod, 2010; Parcerisas, OrtegaGascó, Pujadas, et al., 2021; Winther et al., 2012), and to the control of neural polarization, neurite outgrowth, dendrite development, and synapse formation and maintenance in the cortex and hippocampus through a complex panel of interactors

83 (Leshchyns'Ka et al., 2015; Parcerisas et al., 2020; Sheng et al., 2015; Parcerisas, Ortegagascó, et al., 2021). Interestingly, NCAM2 has been associated with different pathologies including Down syndrome, autism, and Alzheimer's disease (JP et al., 2011; Leshchyns'Ka et al., 2015; Paoloni-Giacobino et al., 1997; Parr et al., 2006; Scholz et al., 2016; Winther et al., 2012). Regarding neurogenesis, Ncam2 has been detected in single-cell RNAseq studies that characterize the genetic profiles of qNSCs and their immediate progeny (Shin et al. 2015; Morizur et al. 2018). However, its role in RGP biology during neurogenesis remains unknown.

In the present study, we characterize the NCAM2 pattern of expression in the adult hippocampal neurogenic area and analyze the role of NCAM2 in the regulation of RGP biology during corticogenesis and in adulthood. To gain further insight into the importance of NCAM2 in the abovementioned processes, we used different biological and genetic tools including hippocampal viral injections, in utero electroporations and in vitro neurosphere cultures. Together, our results indicate that regulated NCAM2 expression levels are crucial for proper adult neurogenesis in addition to its relevant role during brain development. Moreover, we suggest that NCAM2 participates in the fine regulation of quiescency in hippocampal RGPs, a mechanism that could help explaining some pathologies that have been linked to NCAM2 such as Alzheimer's disease which bear a complex phenotype including altered neurogenesis. 


\section{Animals}

105 All experimental procedures were carried out following the guidelines of the Committee

106 for the Care of Research Animals of the University of Barcelona, in accordance with the 107 directive of the Council of the European Community (2010/63 y 86/609/EEC) on animal

108 experimentation. The experimental protocol was approved by the local University 109 Committee (CEEA-UB, Comitè Ètic d’Experimentació Animal de la Universitat de

110 Barcelona) and by the Catalan Government (Generalitat de Catalunya, Departament de

111 Territori i Sostenibilitat).

\section{Antibodies and reagents}

113 The following commercial primary antibodies were used for immunohistochemistry:

114 Anti-ChFP (ab167453, Abcam, 1:300); Anti-DCX (A8L1U, Cell Signaling, 1:500) Anti-GFP 115 (A11122, Invitrogen, 1:2000); Anti-GFAP (Z033401, DAKO, 1:2000); Anti-MAP2 116 (MA1406, Sigma, 1:2000); Anti-NCAM2 (AF778, R\&D Systems, 1:750); Anti-Nestin 117 (MAB353, Chemicon, 1:100), Anti-NeuN (MAB377, Merck, 1:1000); Anti-Sox2 (ab97959, 118 Abcam, 1:500), Anti-Tbr2/EOMES (23345, Abcam, 1:100). Alexa Fluor fluorescent 119 secondary antibodies were from Invitrogen. To counterstain nuclei, the tissue and cells 120 were incubated in 2-(4-amidinophenyl)-1H -indole-6-carboxamidine (DAPI, D-6564, 121 Sigma, 1:1000). Biotinylated-secondary antibodies were from Vector Labs; streptavidin122 biotinylated/HRP complex and ECL were from GE Healthcare. The HRP-labeled 123 secondary antibodies used for western blot were from DAKO. Diaminobenzidine reagent $124(\mathrm{DAB})$ and Eukitt mounting media were from Sigma-Aldrich. Mowiol was from 125 Calbiochem.

\section{Plasmids}

127 The plasmids ShNcam2, pCNcam2.1 and pCNcam2.2 used were described in Parcerisas 128 et al, 2020. The cDNA of Ncam2.1 was amplified from the pCNcam2.1 with 5'129 ACCATGAGCCTCCTCCTCTCC-3' and 5'-CTGACCAAGGTGCTGAAACT-3'and cloned into 130 pWPI (Plasmid \#12254, Addgene) within Pmel site to obtain the pWPI-NCAM2.1. The 131 cDNA of Ncam2.2 was amplified with 5'-ACCATGAGCCTCCTCCTCTCC-3' and 5'132 TCTCTGATCAGGGAGTACCA-3' and cloned into pWPI (Plasmid \#12254, Addgene) within 133 Pmel site to obtain the pWPI-NCAM2.2. 


\section{Production and intrahippocampal injection of retrovirus}

135 The production and intrahippocampal injection of virus was performed as previously

136 described (Parcerisas et al., 2020; Teixeira et al., 2012). Briefly, viral vectors were

137 produced by transient transfection of HEK293T cells with calcium phosphate. Virus were

138 concentrated by ultracentrifugation and resuspended in PBS.

139 For intrahippocampal injections, 8-week-old mice were anaesthetized with

140 ketamine/xylazine mixture and placed on a heating blanket. They were positioned in a

141 Kopf stereotaxic frame and a midline scalp incision was made. The scalp was reflected

142 by hemostats to expose the skull, and bilateral burr holes were drilled. Viruses were

143 then injected (1.5 $\mu$ l of viral stock solution per site) into the left and right dentate gyrus

144 over 20 min using a $5 \mu$ l Hamilton syringe. The micropipette was left in place for an

145 additional $5 \mathrm{~min}$. The coordinates used for the injections (in $\mathrm{mm}$ from Bregma and $\mathrm{mm}$

146 depth below the skull) were as follows: caudal 2.0, lateral 1.6, depth 2.2.

\section{Histological staining and electron microscopy}

148 Animals were anaesthetized and perfused for 20 min with PBS 4\% paraformaldehyde

149 (PFA). The brains were then removed, post-fixed overnight with PBS 4\% PFA, 150 cryoprotected with PBS-30\% sucrose and frozen. Coronal sections $(30 \mu \mathrm{m})$ were

151 obtained with a cryostat and immunohistofluorescence or immunohistochemistry were 152 performed on free-floating sections. Samples were blocked with PBS containing 10\% 153 normal horse serum (NHS) and $0.2 \%$ gelatin; and incubated at $4^{\circ} \mathrm{C}$ overnight with PBS$1545 \%$ NHS primary antibodies. For immunohistofluorescence, sequential incubation was 155 carried out using a secondary antibody (Alexa Fluor, Invitrogen), and the sections were 156 mounted with Mowiol (Calbiochem). The images were acquired with confocal 157 microscopy (Spectral Confocal SP2 Microscope, Leica; Spectral Confocal SP8, Leica and 158 Carl Zeiss LSM880, Zeiss). For immunohistochemistry, sequential incubation was carried 159 out using biotinylated secondary antibodies ( $2 \mathrm{~h}$ at room temperature) and streptavidin160 HRP (1:400; $2 \mathrm{~h}$ at room temperature) was performed in PBS-5\% NGS; bound antibodies 161 were visualized by reaction using $\mathrm{DAB}$ and $\mathrm{H}_{2} \mathrm{O}_{2}$ as peroxidase substrates; the sections 162 were dehydrated and mounted (Eukitt). Images were acquired with AF6000 microscope 163 (Leica) and Olympus Bx61 microscope (Olympus). 
For electron microscopy, sections were cryoprotected in $25 \%$ saccharose and freeze-thawed $(3 x)$ in methylbutane. The sections were then washed in $0.1 \mathrm{M}$ phosphate buffer (PB; $\mathrm{pH} 7.4$ ), blocked in $0.3 \%$ bovine serum albumin-C (BSA), and incubated with a primary chicken anti-GFP antibody (1:200; Aves Labs, Tigard, OR, USA) for $72 \mathrm{~h}$ at $4^{\circ} \mathrm{C}$. The sections were washed in phosphate buffer (PB), blocked in $0.5 \%$ BSA and $0.1 \%$ coldwater fish-skin gelatin (Electron Microscopy Sciences, Hatfield, PA, USA) for $1 \mathrm{~h}$, and subsequently incubated with a colloidal gold-conjugated secondary antibody (1:50; goat anti-chicken IgG gold UltraSmall, Electron Microscopy Sciences) for $24 \mathrm{~h}$ at room temperature. The sections were then washed in PB and $2 \%$ sodium acetate. Silver enhancement (Aurion R-gent Silver enhancer kit, Electron Microscopy Sciences) was performed following the manufacturer's directions, and the sections were washed again in $2 \%$ sodium acetate. To stabilize the silver particles, the samples were immersed in $0.05 \%$ gold chloride (Sigma) for $10 \mathrm{~min}$ at $4^{\circ} \mathrm{C}$, washed in sodium thiosulfate, washed in $\mathrm{PB}$, and then postfixed in $2 \%$ glutaraldehyde for $30 \mathrm{~min}$. The sections were incubated in $1 \%$ osmium tetroxide and $7 \%$ glucose and then washed in deionized water. Subsequently, sections were partially dehydrated in $70 \%$ ethanol and incubated in $2 \%$ uranyl acetate in $70 \%$ ethanol in the dark for $2.5 \mathrm{~h}$ at $4^{\circ} \mathrm{C}$. Brain slices were further dehydrated in ethanol followed by propylene oxide and infiltrated overnight in Durcupan ACM epoxy resin (Fluka, Sigma-Aldrich, St. Louis, USA). The following day, fresh resin was added, and the samples were cured for $72 \mathrm{~h}$ at $70^{\circ} \mathrm{C}$. Following resin

184 hardening, 1.5- $\mu \mathrm{m}$ semi-thin sections were selected under light microscopy based on their immunolabeling and detached from glass-slides by repeated freezing and thawing in liquid $\mathrm{N}_{2}$. Ultra-thin sections were obtained at 60-70 $\mathrm{nm}$ from selected semi-thin sections. Photomicrographs were obtained using a FEI Tecnai $G^{2}$ Spirit (FEI Europe, Eindhoven, Netherlands) using a digital camera Morada (Olympus Soft Image Solutions $\mathrm{GmbH}$, Münster, Germany).

\section{In utero electroporation}

191 In utero microinjection and electroporation were performed at E14.5 as described (Simó 192 et al. 2010; Parcerisas et al. 2020b), using timed pregnant CD-1 mice (Charles River 193 Laboratories). Briefly, DNA solutions were mixed in $10 \mathrm{mM}$ Tris ( $\mathrm{pH} \mathrm{8.0)}$ with $0.01 \%$ Fast 194 Green. Needles for injection were pulled from Wiretrol II glass capillaries (Drummond 
195 Scientific) and calibrated for $1 \mu$ injections. Forceps-type electrodes (Nepagene) with 5-

$196 \mathrm{~mm}$ pads were used for electroporation (five 50-msec pulses of $45 \mathrm{~V}$ at E14.5). Brains

197 were collected at E19.5/P0 or P5, dissected, and successful electroporations identified

198 by epifluorescence microscopy. Positive brains were fixed in $4 \%$ formalin in $0.1 \mathrm{M}$

199 phosphate buffer saline (PBS) and cryoprotected in $30 \%$ sucrose/PBS overnight at $4^{\circ} \mathrm{C}$.

200 Brains were frozen in O.C.T compound before fourteen-micrometer-thick brain cross-

201 sections were obtained with cryostat and placed on slides. Sections were antigen-

202 retrieved by immersion of the slides in $0.01 \mathrm{M}$ sodium citrate buffer, $\mathrm{pH} 6.0$ at $95^{\circ} \mathrm{C}$ for

$20320 \mathrm{~min}$. Sections were blocked for $2 \mathrm{~h}$ with $10 \%$ normal goat serum, $10 \mathrm{mM}$ glycine, and

$2040.3 \%$ Triton X-100 in PBS at room temperature. Primary antibodies (anti-GFP and anti-

205 ChFP) were incubated overnight at $4^{\circ} \mathrm{C}$. Slides were washed four times for $10 \mathrm{~min}$ in

206 0.1\% Triton X-100/PBS. Secondary antibodies were added for $2 \mathrm{~h}$ at room

207 temperature and the slides were washed as before and coverslipped with Prolong

208 Gold anti-fade reagent (Molecular Probes). Most images were obtained with

209 epifluorescent illumination and a 10x objective (Leica 760 or AF6000). Positions of

210 GFP- or ChFP-positive neurons were recorded from several sections per embryo. Data

211 were collected from the lateral part of the anterior neocortex. For a BIN10

212 quantification, the cortex was divided into 'BINs' as follows: the distance from the pial

213 surface to the bottom of the SVZ was measured and divided into 10 equal-sized BINs.

214 The percentage of GFP- or ChFP-labeled neurons in each BIN for each embryo was

215 then calculated. Graphs plot the mean and standard error of \% neurons in each BIN for

216 the $\mathrm{N}$ embryos in a group.

217 Neurospheres culture

218 Neurospheres cultures were derived from 7-8 postnatal day (P7-P8) mice following the

219 modified protocol described by Walker \& Kempermann, 2014. Briefly, the SVZ of the

220 lateral ventricles and the SGZ of the hippocampus were dissected in PBS. After trypsin

221 (GIBCO) and DNAse (Roche diagnostic) treatments, the tissue was dissociated with

222 gentle sweeping. Cells were counted and plated in non-adherent 24 well plates in

223 Neurobasal medium containing 2\% B27 supplement (GIBCO), penicillin/streptomycin

224 (Life technologies) and Glutamax (Life technologies), $20 \mathrm{ng} / \mathrm{ml} \mathrm{EGF,} 20 \mathrm{ng} / \mathrm{ml} \mathrm{bFGF}$ and 
$2252 \mu \mathrm{g} / \mathrm{ml}$ heparin. Cells were incubated at $37^{\circ} \mathrm{C}$ with $5 \% \mathrm{CO}_{2}$ and subcultured every $2-3$

226 days.

227 For the growth analysis, neurospheres from the SGZ were dissociated with

228 trypsin and infected at passage 2 with viruses (pWPI, pWPI-NCAM2.1, pWPI- NCAM2.2,

229 ShNcam2 or ShCnt). GFP positive cells were selected by flow cytometry (BD FACSAria

230 Fusion), plated in non-adherent 24-well plates and analyze during 5 consecutive days.

231 High content image acquisition was performed with an Automated Wide-field Olympus

232 IX81 Microscope (Olympus Life Science Europe, Waltham, MA) and a 4x UPlan FL N

233 objective. ScanR Acquisition software version 2.3.0.5 was used to automatically record

234 adjacent fields of view taking $20(5 \times 4)$ z-stacks (8 slices with a z-step of $200 \mathrm{~nm}$ ) per

235 well, with $10 \%$ of overlap to enable automatic image stitching. Neurosphere size was

236 quantified by means of 3 different Fiji macros. In brief, tailor-made macros were used

237 to project each z-stack, to stitch these projections and to quantify the size of each

238 neurosphere.

239 Image analysis

240 All images were processed and quantified using the ImageJ software (NIH).

241 Statistical analysis

242 Statistical analysis was carried out using the Prism 8 software. Significance between two

243 experimental groups was analysed using the unpaired Student's $t$-test. Differences

244 between groups in distribution of cells in corticogenesis were assessed by two-way

245 ANOVA followed by Bonferroni's comparison post hoc test. To determine differences

246 between more than two groups in the adult neurogenesis characterization experiments,

247 one way ANOVA was used. Post-hoc comparisons were performed by Tukey's test and

248 significance level was set at $P>0.05$ : ${ }^{*} P<0.05, * * P<0.01$, and ${ }^{*} * P<0.001$. To determine

249 differences between two groups, Student's t-test and significance level was set at

$250 P>0.05:{ }^{*} \mathrm{P}<0.05,{ }^{*} \mathrm{P}<0.01$, and ${ }^{* * *} \mathrm{P}<0.001$. Statistical values are presented as mean \pm

251 standard error of the mean (SEM). 


\section{RESULTS}

253 Differential expression pattern of NCAM2 in the dentate gyrus

254 Since cell adhesion molecules are important structural elements of the neurogenic 255 niches we first characterize the expression of NCAM2 in the different populations of cells 256 at the DG of P45 mice by immunofluorescence. Dentate RGPs undergo several 257 morphological and electrophysiological changes while expressing different markers 258 through the neurogenic process to finally give rise to mature neurons. To identify type I progenitors we used the GFAP/Sox2 or Nestin markers while Tbr2 was selected to mark

260 type II proliferative progenitors (Kempermann et al., 2015). In addition, we detected 261 neuroblasts or immature neurons with antibodies against DCX; and mature neurons 262 labelling NeuN. As NCAM2 is a membrane protein, the general pattern of NCAM2 263 staining show clear staining in the delineating cells bodies and the dendrites of neurons. 264 Confocal microscopy analysis reveal strong NCAM2 signal in GFAP/Sox2 or Nestin positive cells with the typical morphology of type I progenitors (i.e: triangular cell body

266 located in the SGZ and a unique dendrite extended into the molecular layer) (Fig. 1A-B). 267 Contrariwise, images suggest that NCAM2 expression in Tbr2 positive cells is low, 268 although it is difficult to determine the expression of NCAM2 and Tbr2 in the same cells 269 due to the localization of both proteins (Fig. 2C). Among the DCX positive cells 270 population, we found different phenotypes with differences in NCAM2 staining. While 271 some DCX positive cells display faint or undetected NCAM2 staining, other cells present 272 higher levels of the protein (Supplementary Fig. 1A). Lastly, mature granule cells that 273 express NeuN also present NCAM2 labelling, as expected (Supplementary Fig. 1B). Therefore, the characterization of the expression pattern of NCAM2 in the 275 dentate gyrus of the hippocampus suggests a differential expression of the protein in 276 the main actors of the neurogenic process: while both RGPs and mature neurons express appreciable NCAM2 staining the intermediate type II-III progenitors may have a minimum in NCAM2 expression. 


\section{NCAM2 modulates adult neurogenesis in the hippocampus}

280 With the purpose to study the potential role of NCAM2 in adult neurogenesis, we

281 modulate the expression of the NCAM2 protein in the hippocampal neurogenic region.

282 We stereotaxically injected transduced the DG of 8 week-old mice with

283 NCAM2.1/NCAM2.2-overexpressing or ShNCAM2-silencing lentiviruses, which bear

284 preferential infectivity on progenitor cells or neuroblasts. We analyzed the transduced

285 DGs 4 weeks after surgery. Mice injected with control viruses exhibited the 286 characteristic morphology of dentate granule cells (i.e. round soma in the granule cell

287 layer, and apical dendrites ramifying in the molecular layer and covered by dendritic

288 spines) (Fig.2A, first panel). Similar results were found in mice injected with ShNCAM2

289 viruses, indicating that the downregulation of NCAM2 does not alter the formation,

290 survival, or maturation of new adult-born neurons in the DG. (Fig. 2A, second panel).

291 Conversely, we found that many cells infected with NCAM2.1 and NCAM2.2

292 overexpressing viruses did not exhibit the typical morphology of maturing granule cells

293 but a RGP-like phenotype (i.e. triangular cell bodies located in the inner GL, with a

294 unique, short radial process spanning the GL and ramifying profusely in the inner

295 molecular layer) (Fig. 2A, third and fourth panel). Some infected cells, however,

296 resembled type II progenitors or neuroblasts (i.e. irregular soma with short processes

297 oriented tangentially or rounded soma with a short apical dendrite oriented towards the

298 molecular layer) or immature granule cells. Enrichment in RGP-like phenotype

299 apparently was more prominent upon NCAM2.2-overexpression.

300 To further characterize the phenotype of NCAM2 overexpressing cells, we

301 performed fine structure analysis of GFP-labelled cells, identified by GFP-immunogold

302 electron microscopy (Fig. 2B). Confirming our optical microscopy results, most control

303 infected cells at the injection site corresponded to dentate granule cells which were

304 closely apposed in the granule layer (GL). These cells showed a typical round-shaped

305 soma, most of it occupied by the nucleus, which displayed chromatin aggregates. The

306 cytoplasm was comprised by a thin space with a few long cisternae of endoplasmic

307 reticulum and abundant free ribosomes. Nevertheless, we also observed GFP-positive

308 cells in the SGZ. Among them, we identified RGPs and type II cells or neuroblasts. As

309 previously described (Seri et al. 2004), RGPs were recognized as cells with a large cell 
310 body with a major radial process that penetrates the granular layer extending thin

311 lateral processes between granule neurons. They present a round or triangular nucleus,

312 electron lucent cytoplasm, irregular contour and intermediate filaments in their

313 cytoplasm. On the other hand, type II cell (or neuroblast) features include a smooth

314 contour, dark scant cytoplasm, abundant polyribosomes and a less developed

315 endoplasmic reticulum than granule cells. Interestingly, NCAM2.1/NCAM2.2-

316 overexpressing GFP-positive cells were mainly detected in the SGZ and, according to

317 their fine structure, could be identified RGPs (Fig. 2B).

318 Cell autonomous overexpression of NCAM2 retains adult-born DG cells in a RGP-like 319 phenotype

320 To better understand the events triggered by the expression of NCAM2 isoforms, 321 animals injected with control and NCAM2 overexpressing viruses were sacrificed at 322 different time points including earlier stages ( 3 days, 1 week, 2 weeks and 4 weeks) (Fig.

323 3A). As a starting point, animals were sacrificed 3 days after injection. Although the 324 infection is not strictly restricted to progenitor cells, as expected, the majority of the 325 infected cells exhibit a RGPs morphology 3 days post-injection in all the experimental 326 conditions (Consiglio et al. 2004; Jandial et al. 2008) (Fig. 3B). Focusing on posterior 327 time points, most cells infected with control vectors at 1 week post-injection showed a 328 morphology typical of immature granule cells that appeared progressively more mature 329 at 2 and 4 weeks post-injection. In contrast, the shapes of NCAM2.1- and NCAM2.2330 overexpressing cells remained constant overtime, with most of the labeled cells 331 exhibiting an RGP-like cell morphology, while others exhibited intermediate 332 progenitors- or neuroblast-like phenotypes (Fig. 3B).

The phenotype of cells infected with the viral vectors was additionally 334 characterized evaluating the expression of specific cell markers at the different time points analyzed. The triple immunostaining of GFP/Sox2/GFAP was used to determine 336 the proportion of RGPs within the pool of infected cells (Fig. 4A-B). At 3 days after 337 injection most of control-infected cells were Sox2/GFAP double positive (Fig. 4C). 338 Similarly, also NCAM2.1- and NCAM2.2-infected cells were mostly positive for both 339 markers at 3 days post infection (Fig. 4C). Analyzing the evolution of GFP-/Sox2-/GFAP340 positive progenitors in the control conditions we observed a significant and progressive 
341 decline in the number of progenitors over time (Fig. 4C-D). In contrast, in the animals

342 infected with NCAM2.1 or NCAM2.2 overexpressing viruses, we noticed a much less

343 marked decrease in the proportion of those progenitors along the time-course, thus

344 suggesting an arrest of the cells in the progenitor stage (Fig. 4C-D). We confirmed that

345 most NCAM2.1- and NCAM2.2-overexpressing cells morphologically characterized as

346 neuronal progenitor cells expressed the neuronal progenitor markers GFAP and Sox2 at

3471 week after injection (Fig. 4A,C). Additionally, quantification of the percentage of

348 GFP/Sox2/GFAP revealed a maintenance of high proportion of GFAP/Sox2 positive cells

349 in both NCAM2.1 and NCAM2.2 overexpressing conditions also at 2 weeks and 4 weeks,

350 being more pronounced in the case of NCAM2.2 isoform (Fig. 4C).

351 The impact of NCAM2 overexpression in the process of neurogenesis was

352 complemented quantifying the number of DCX positive cells at 2 and 4 weeks after viral

353 transduction (Fig. 5A). According to the expected evolution of the neurogenic events,

354 we observed a high percentage of DCX positive cells at 2 weeks after injection followed

355 by a decrease at 4 weeks (Fig. $\mathbf{5 C}$ ). The above-mentioned decline is not detected when

356 NCAM2.1 or NCAM2.2 are overexpressed and we found a persisting number of DCX

357 positive cells from 2 to 4 weeks after transduction. Finally, the number of NeuN mature

358 neurons 4 weeks post-injection was also analyzed (Fig. 5B). In agreement with the

359 previous data, we found a trend to show reduced percentages of NeuN positive neurons

360 in the overexpression conditions at 4 weeks post-induction, reaching statistically

361 significance for the NCAM2.2 isoform compared to controls (Fig. 5D).

362 This time-course analysis suggests that the observations at 4 weeks post-

363 injection time on overexpression of NCAM2 are not attributable to a de-differentiation

364 of immature neurons, but rather to a temporarily arrest of the RGP-like phenotype in

365 the SGZ that leads to a delay in the formation of new neurons. 


\section{NCAM2 overexpression do not arrest embrionary RGPs}

367 Since our results point out to an important role of NCAM2 in the regulation of adult RGPs

368 and NCAM1 is involved both in adult and embryonic neurogenesis (Angata et al. 2007b;

369 Boutin et al. 2009), we next sough to study the potential role of NCAM2 in RGPs during

370 embryonic stages. We performed in utero electroporation experiments using isoform-

371 specific overexpressing vectors (i.e. NCAM2.1 and NCAM2.2). Embryos were

372 electroporated at E15 (using GFP or ChFP as reporter genes) and brains were analysed

373 at P0 and P5, by counting the distribution of electroporated neurons across cortical

374 layers. Interestingly, we found a moderate non-significant of cells located in the

375 neurogenic areas, VZ and the intermediate zone (IZ), in cortices electroporated with

376 NCAM2 isoforms (Fig. 6A). However, we observed alterations in the migration of

377 neurons when modulating NCAM2 expression. Our previous study (Parcerisas et al.,

378 2020) showed that both NCAM2 isoforms are expressed in the developing cortex and

379 that its expression is necessary for correct neuronal migration, since NCAM2 knock-

380 down leads to neuronal mispositioning. In the present analysis, we observed that at P0, most E15-born control neurons were present in the upper portion of the cortical plate and displayed a typical immature pyramidal neuron shape, with a main apical dendrite

383 directed towards the marginal zone (Fig. 6A-E). In the case of E15-born NCAM2.2overexpressing neurons, we observed an altered distribution with a significant reduction of neurons in the upper portion of the cortical plate (Bin 10) (Fig. 6A-B). E15-born NCAM2.1-overexpressing neurons also had a tendency to allocate below bin 10 (Fig. 6AB). A synergistic effect was found when embryos were electroporated with both isoforms (NCAM2.1+NCAM2.2) simultaneously (Fig. 6A-B). Additionally, in contrast with NCAM2 depletion, NCAM2 overexpression apparently does not disrupt normal dendritic arborization at this stage.

In contrast, at P5, E15-electroporated neurons displayed a similar distribution in

392 both for control and NCAM2-overexpressing conditions, with most neurons being

393 located in the lower part of layer II-III (Fig. 6C-D). Our results suggest that NCAM2.1 and

394 NCAM2.2 overexpression statistically not affect the proliferation, survival and 395 differentiation of RGPs during embryonic stages but leads to transient migratory deficits.

396 NCAM2 expression levels affect the growth of hippocampal-derived neurospheres 
397 The implications of NCAM2 in adult neurogenesis were further investigated in vitro using 398 neurospheres. Hippocampal NSCs were obtained from P6/7 mice and grown as 399 neurospheres in medium containing EGF and bFGF. Neurospheres were dissociated and

400 cells were infected with Control, ShNCAM2, NCAM2.1, or NCAM2.2-overexpressing 401 viruses all of them co-expressing GFP as a reporter gene. GFP-positive cells were 402 selected by flow cytometry, plated in 24 well plates and analyzed by ScanR microscopy

403 to measure the individual area of a total of 100-300 growing neurospheres per condition 404 during 5 consecutive days (Fig. 7A). Whereas the downregulation of NCAM2 led to the 405 formation of larger neurospheres, compared to controls, neurospheres derived from 406 NCAM2.1- or NCAM2.2-overexpressing cells tended to be smaller (Fig. 7B-C). Focusing 407 the analysis on day 3, we observed a different distribution of the neurospheres 408 according to their area. The descriptive analysis of the frequency distributions shows 409 that the mean and median values of the distribution are lower in the NCAM2.1 and 410 NCAM2.2 overexpressing neurospheres than in controls; and higher in the ShNCAM2 411 condition (Fig. 7D-E).

412 These findings further support the notion that NCAM2.1 and NCAM2.2 are 413 involved in the regulation of NSCs proliferation. 
415 The present work provides a deeper understanding on the relevant functions of NCAM2

416 during embryonic development and adult neurogenesis. Our results suggests that

417 NCAM2 levels regulate the RGP-to-immature neuron transition in the adult DG. In

418 contrast, our data indicate that correct NCAM2 levels are not necessary for cortical

419 neurogenesis, but relevant for cortical migration.

420 The injection of lentivirus to modulate the expression of NCAM2 in the 421 progenitor cells of the SGZ in the hippocampus reveals a compelling role of NCAM2 in 422 the regulation of neural progenitors. While the depletion of NCAM2 had minor effects, 423 the overexpression of NCAM2 seems to arrest cells into an RGP-like phenotype and 424 delay the formation of new granule cells, as characterized by morphology, 425 immunohistochemical markers, and ultrastructure. However, when analyzing the 426 effects of NCAM2 overexpression in the regulation of embryonic RGPs, we did not find 427 clear evidences of any alterations in the survival, proliferation or differentiation of 428 progenitor cells. We found that NCAM2 upregulation results in an early and transiently 429 altered neuron distribution, suggesting a delay in their migration during cortical 430 development. Our previous results also showed that the downregulation of NCAM2 led 431 to an alteration of cortical migration leading to mislocalization of layer II-III fated 432 neurons and altered morphology (Parcerisas et al., 2020). Neuronal migration is a key 433 process in corticogenesis, the disruption of which is associated to many diseases 434 including autism and schizophrenia (Hussman et al., 2011; Petit et al., 2015; Scholz et 435 al., 2016). The mechanism underlying the effects of NCAM2 are not known. The 436 interaction of NCAM2 with microtubule-associated proteins, such as MAP1B, that also 437 participate in the regulation of neuronal migration has also been described (González438 Billault et al., 2005; Kawauchi \& Hoshino, 2008; Parcerisas et al., 2020; Parcerisas, 439 Ortega-gascó, et al., 2021). roles of NCAM2 during adult and embryonic stages. In spite of the embryonic origin of adult RGPs, adult and embryonic progenitors are subject to distinct regulation (Urbán and Guillemot 2014; Berg et al. 2018; Daniel Berg et al. 2019). While embryonic RGPs have a highly proliferative rate necessary for the rapid growth of neural tissues (Urbán 
et al., 2019; Urbán 2014); adult RGPs are mostly found in a quiescent state, a mitoticdormant phase with a low rate of metabolic activity but with a high sensitivity to environment signals (Urbán et al., 2019). The quiescence of RGPs is actively maintained and the regulation of the transition from quiescence to activation is crucial to preserve a pool of RGPs throughout life. Adult RGPs are found in neurogenic niches, specialized microenvironments composed by different cellular types, ECM molecules, soluble factors and cell surface molecules (Bian, 2013). Neurogenic niches are crucial for the regulation of RGPs properties and to maintain the quiescence/activation balance (Llorens-Bobadilla and Martin-Villalba, 2017; Basak et al., 2018; Kalamakis et al., 2019) as they convey the different physiological stimuli (Fabel and Kempermann 2008; Wang et al. 2011; N and F 2014; Ding et al. 2020) that induce the activation of quiescent RGPs. important for sustaining the architecture of the niche but also participate in signal transduction regulating stem cells, survival, proliferation, migration or differentiation. As a matter of fact, different cell adhesion molecules such as cadherin/protocadherins, VCAM1, L1CAM or NCAM1 have been identified playing a distinct role in the neurogenic niches (K. Angata et al., 2007; Bian, 2013; Boldrini et al., 2018; Bonfanti, 2006; Dihné et al., 2003; Karpowicz et al., 2009; Marthiens et al., 2010; Morante-Redolat \& Porlan, 2019; Morizur et al., 2018; Shin et al., 2015). Specifically, it has been described that cell adhesion molecules could be important regulators of the quiescence/activation balance. The genetic profiles of RGPs showed an enriched expression of genes involved in cellmicroenvironment interaction and cell-cell adhesion, and genes linked to cell membrane

467 (Artegiani et al., 2017; Basak et al., 2018; Ding et al., 2020; Dulken et al., 2017; Hochgerner et al., 2018; Llorens-Bobadilla et al., 2015; Morizur et al., 2018; Shin et al., 2015). Upon activation, RGPs proliferate and progress to rapid amplifying intermediate progenitors or type II cells. A decrease in the expression of some cell adhesion molecules seems to be necessary for the activation of quiescent RGPs, their transition to intermediate progenitors and the proliferation of these progenitors (Morizur et al., 2018; Shin et al., 2015; Codega et al., 2014; Xie et al., 2020). A similar expression pattern

474 has been presented in this study when immunodetecting NCAM2 in the SGZ populations. The proposed pattern of NCAM2 expression along dentate neurogenesis 
476 cell types, supported by single cell RNA (Shin et al., 2015), confirms high NCAM2

477 expression in type I progenitors and low levels in intermediate progenitors. In fact, the

478 expression pattern of Ncam2 gene during the early neurogenic events is similar to other

479 genes related to the maintenance of stem cells quiescence (e.g: NPas3 or Aqp4) (Shin et

480 al., 2015; Urbán et al., 2019) presenting high levels of expression in qNSCs that

481 progressively decrease during their activation and transition to intermediate

482 progenitors (Shin et al. 2015; Morizur et al. 2018) (Supplementary Fig. 2, Fig. 9). Once

483 the precursor cell phase is completed, the levels of NCAM2 seem to experiment a

484 progressive increase in the newborn DCX positive maturing neurons reaching high levels

485 of expression in NeuN neurons. The increase of NCAM2 could be explained by the

486 relevance of the protein for dendrite development, axon formation and synaptogenesis

487 (Alenius \& Bohm, 2003; Kulahin \& Walmod, 2010; Winther et al., 2012, Parcerisas et al., 488 2020).

489 The levels of NCAM2 seems to be important for the regulation of RGPs 490 behaviour. In fact, our data show how changes in NCAM2 levels modifies the normal course of the neurogenic events. The upregulation of NCAM2 dramatically decrease the generation of newborn neurons. Diverse underlying mechanisms could explain these

493 findings. The upregulation of NCAM2 could affect the survival of the newborn cells, 494 induce the de-differentiation of developing neurons or either alter the differentiation of 495 the newborn neurons. However, considering the expression pattern of the protein and 496 the relevance of cell adhesion molecules in the regulation of RGPs (Codega et al. 2014; 497 Morizur et al. 2018; Xie et al. 2020), our main hypothesis is that NCAM2 is important for 498 the regulation and maintenance of RGPs quiescence. Considering that the 499 overexpression of NCAM2 induces the retention of progenitor cells into a RGP state, we 500 should expect that the downregulation of the protein promote the activation of RGPs to 501 increase proliferation. In contrast, after inducing NCAM2 depletion in the hippocampus 502 of injected mice, we did not detect an increase in the number of newly produced 503 neurons. The underlying cause for this inconsistency might rest on the limitations 504 imposed by the lack of uniformity in the infection of cells, preventing quantitative 505 analyses of the number of new neurons generated. In order to overcome these 506 limitations, we further investigated the effect of NCAM2 in vitro using a neurosphere 507 assay. We observed that the downregulation of NCAM2 expression in progenitor cells in 
508 vitro increases the growth of neurospheres while overexpression of NCAM2 isoforms 509 decreases the area of the neurospheres. The effects of NCAM2 in the proliferation of 510 NSCs in vitro has previously been observed in progenitor cells that form the spinal cord

511 (Deleyrolle et al. 2015) and supports the data obtained in the present study.

Taking these results together, we postulate that the regulation of NCAM2 513 expression levels is necessary for the maintenance of RGPs quiescence and the 514 activation of proliferation. High levels of NCAM2 arrest cells in a quiescent state while 515 the downregulation of ncam2 allows RGPs to exit quiescence and enter the cell cycle to 516 proliferate and differentiate (Fig. 9). The temporary retention of cells in the progenitor 517 stages would led to a delay in the neurogenic events postponing the generation and 518 maturation of granule cells although other explanations may contribute (e.g. changes in 519 cell survival or differentiation to other cell types). Further research is needed to 520 understand the mechanisms by which NCAM2 regulates RGPs quiescence, cell 521 proliferation, and differentiation in adulthood. One hypothesis is that NCAM2 could 522 interact with growth factor receptors such as the epidermal growth factor receptor 523 (EGFR) or the fibroblast growth factor receptor (FGFR). Growth factors are important 524 regulators of the activation of quiescent RGPs; for example, active RGPs in the SVZ could 525 be identified by the expression of EGFR (Aguirre et al., 2010; Urbán et al., 2019). It has 526 been described that NCAM2 binds to FGFR and EGFR (Deleyrolle et al. 2015; Rasmussen 527 et al. 2018), and the interaction of other cell adhesion molecules, such as L1CAM or 528 NCAM1, with FGFR has also been reported (Kulahin et al. 2008; Francavilla et al. 2009). 529 Moreover, it has been shown that the overexpression of NCAM1 reduces baseline levels 530 of EGFR, enhancing the EGF-induced receptor down-regulation, and that the depletion 531 of NCAM2 increases the levels of the ErbB2 growth factor receptor (Povlsen et al. 2008;

532 Deleyrolle et al. 2015). Another possibility is that NCAM2 expression could cause 533 cytoskeletal rearrangements, which are known to influence the neurogenetic process 534 (Compagnucci et al., 2016; Parcerisas et al., 2020; Parcerisas, Ortega-gascó, et al., 2021). Neurogenic niches are complex microenvironments where RGPs receive and 536 interact with multiple signals. Cell adhesion molecules are key elements for the 537 transduction of the signals and the regulation of stem cells behavior. Our work provides 538 evidence for a significant function of NCAM2 in the regulation of RGPs during adult 
539 neurogenesis. Furthermore, we reveal the importance of NCAM2 expression in the

540 regulation of neuronal migration and differentiation during the corticogenesis process

541 in the embryonic development. Overall, the present study contribute to a better

542 understanding of the implications of NCAM2 during neuronal development and adult 543 plasticity. 


\section{CONFLICT OF INTEREST}

545 The authors declare no competing financial interests.

\section{AUTHORS CONTRIBUTION}

547 E.S., L.P., and A.P. conceived and designed the study. A.O-G. and A.P. performed most

548 of the experiments and analyzed data. K.H. and S.S. designed and performed in 549 utero electroporation. V.H-P. and J.M.G-V. designed and produced the electron 550 microscopy experiments and analysis. F.U. supervised the RGP characterization. A.E-T 551 and M.B participate in some experiments. A.O-G., A.P., V.H.-P., L.P., and E.S. wrote the 552 manuscript. All authors read and corrected the manuscript.

\section{FUNDING}

554 This work was supported by grants from the Spanish Ministry of Science, Innovation and 555 Universities to V.H-P. (PCI2018-093062) and to E.S and L.P. (PID2019-106764RB-C21/ $556 \mathrm{AEI} / 10.13039 / 501100011033)$, from the Spanish Ministry of health (ISCIII-CIBERNED), 557 from The Secretary of Universities and Research of the Department of Economy and 558 Knowledge of the Generalitat de Catalunya to A.P, from the Valencian Council for 559 Innovation, Universities, Science and Digital Society (PROMETEO/2019/075) to J.M.G-V, 560 and from the National Institute of Health (NIH) (R01NS109176) to S.S..

\section{ACKNOWLEDGEMENTS}

562 We thank A. Lladó and S. Tosi (microscopy facility of the IRB-Barcelona) for FIJI macro

563 design and technical assistance in ScanR acquisition; L. Bardia (microscopy facility of the 564 IRB-Barcelona) for support and technical assistance; E. Coll and M. Calvo for technical 565 assistance (microscopy facilities of the University of Barcelona). Marta Pérez for 566 technical support in viral production (Universitat Internacional de Catalunya). The 567 members of the Department of Cell Biology, Physiology and Immunology (University of 568 Barcelona), specially J. Correas for cryostat support and Esther Verdaguer for valuable 569 support; and members of the Soriano lab for experimental help and comments. 


\section{ABBREVIATIONS}

571

$\begin{array}{ll}\text { CAM } & \text { Cell adhesion molecules } \\ \text { CNS } & \text { Central nervous system } \\ \text { DAB } & \text { Diaminobenzidine } \\ \text { DAPI } & \text { 2-(4-amidinophenyl)-1H -indole-6-carboxamidine } \\ \text { DG } & \text { Dentate gyrus } \\ \text { EGF } & \text { Epidermal growth factor } \\ \text { EGFR } & \text { Epidermal growth factor receptor } \\ \text { FGF } & \text { Fibroblast growth factor } \\ \text { FGFR } & \text { Fibroblast growth factor receptor } \\ \text { GFAP } & \text { Glial Fibrillary acidic protein } \\ \text { GFP } & \text { Green Fluorescent Protein } \\ \text { GL } & \text { Granule layer } \\ \text { GPI } & \text { Glycosylphosphatidylinositol } \\ \text { H } & \text { Hilus } \\ \text { HRP } & \text { Horseradish peroxidase } \\ \text { IZ } & \text { Intermediate zone } \\ \text { L1CAM } & \text { L1 cell adhesion molecule } \\ \text { MAP2 } & \text { Microtubule-associated protein 2 } \\ \text { ML } & \text { Molecular layer } \\ \text { MTT } & \text { (3-(4,5-dimethylthiazol-2-yl)-2,5- diphenyltetrazolium bromide } \\ \text { NCAM1 } & \text { Neural cell adhesion molecule 1 } \\ \text { NCAM2 } & \text { Neural cell adhesion molecule 2 } \\ \text { NGS } & \text { Normal goat serum } \\ \text { NHS } & \text { Normal horse serum } \\ \text { NSC } & \text { Neural stem cell } \\ \text { PB } & \text { Phosphate buffer } \\ \text { PBS } & \text { Phosphate buffer saline } \\ \text { PFA } & \text { Paraformaldehyde } \\ \text { RGP } & \text { Radial glial progenitor } \\ \text { SGZ } & \text { Subgranular zone } \\ \text { Sox2 } & \text { Sry-related HMG box transcription factor } \\ \text { SVZ } & \text { Subventricular zone } \\ \text { VCAM1 } & \text { Vascular cell adhesion molecule 1 } \\ \end{array}$




\section{REFERENCES}

574 Aguirre A, Rubio ME, Gallo V. 2010. Notch and EGFR pathway interaction regulates neural stem cell number and self-renewal. Nature. 467:323-327.

Alenius M, Bohm S. 2003. Differential function of RNCAM isoforms in precise target selection of olfactory sensory neurons. Development. 130:917-927.

Altman J, Das GD. 1965. Post-natal origin of microneurones in the rat brain. Nature. 207:953-956.

Angata K, Huckaby V, Ranscht B, Terskikh A, Marth JD, Fukuda M. 2007. Polysialic AcidDirected Migration and Differentiation of Neural Precursors Are Essential for Mouse Brain Development. Mol Cell Biol. 27:6659-6668.

Artegiani B, Lyubimova A, Muraro M, van Es JH, van Oudenaarden A, Clevers H. 2017. A Single-Cell RNA Sequencing Study Reveals Cellular and Molecular Dynamics of the Hippocampal Neurogenic Niche. Cell Rep. 21:3271-3284.

Basak O, Krieger TG, Muraro MJ, Wiebrands K, Stange DE, Frias-Aldeguer J, Rivron NC, Troy+ brain stem cells cycle through quiescence and regulate their number by sensing niche occupancy. Proc Natl Acad Sci U S A. 115:E610-E619.

Berg DA, Bond AM, Ming G, Song H. 2018. Radial glial cells in the adult dentate gyrus: what are they and where do they come from? F1000Research. 7.

Bergmann O, Spalding KL, Frisén J. 2015. Adult neurogenesis in humans. Cold Spring Harb Perspect Med. 5.

Bian S. 2013. Cell Adhesion Molecules in Neural Stem Cell and Stem Cell- Based Therapy for Neural Disorders. In: Neural Stem Cells - New Perspectives. InTech.

Boldrini M, Fulmore CA, Tartt AN, Simeon LR, Pavlova I, Poposka V, Rosoklija GB, Stankov A, Arango V, Dwork AJ, Hen R, Mann JJ. 2018. Human Hippocampal Neurogenesis Persists throughout Aging. Cell Stem Cell. 22:589-599.e5.

Bonfanti L. 2006. PSA-NCAM in mammalian structural plasticity and neurogenesis. Prog Neurobiol.

Boutin C, Schmitz B, Cremer H, Diestel S. 2009. NCAM expression induces neurogenesis in vivo. Eur J Neurosci. 30:1209-1218. 
Compagnucci C, Piemonte F, Sferra A, Piermarini E, Bertini E. 2016. The cytoskeletal

Consiglio A, Gritti A, Dolcetta D, Follenzi A, Bordignon C, Gage FH, Vescovi AL, Naldini L. 2004. Robust in vivo gene transfer into adult mammalian neural stem cells by lentiviral vectors. Proc Natl Acad Sci. 101:14835-14840.

Daniel Berg AA, Su Y, Jimenez-Cyrus D, Ming G-L, Song H, Bond Correspondence AM,

Denoth-Lippuner A, Jessberger S. 2021. Formation and integration of new neurons in the adult hippocampus. Nat Rev Neurosci. 1-14.

Dihné M, Bernreuther C, Sibbe M, Paulus W, Schachner M. 2003. A new role for the cell

Ding WY, Huang J, Wang H. 2020. Waking up quiescent neural stem cells: Molecular mechanisms and implications in neurodevelopmental disorders. PLoS Genet. 16:126.

Fabel K, Kempermann G. 2008. Physical activity and the regulation of neurogenesis in the adult and aging brain. NeuroMolecular Med.

634 Francavilla C, Cattaneo P, Berezin V, Bock E, Ami D, De Marco A, Christofori G, Cavallaro 
637 Gage FH. 2019. Adult neurogenesis in mammals. Science (80- ). 364.

638 Ghosh HS. 2019. Adult Neurogenesis and the Promise of Adult Neural Stem Cells. J Exp $639 \quad$ Neurosci.

640 Gonçalves JT, Schafer ST, Gage FH. 2016. Adult Neurogenesis in the Hippocampus: From $641 \quad$ Stem Cells to Behavior. Cell.

642 González-Billault C, Del Río JA, Ureña JM, Jiménez-Mateos EM, Barallobre MJ, Pascual 643 M, Pujadas L, Simó S, Torre A La, Gavin R, Wandosell F, Soriano E, Ávila J. 2005. A 644 role of MAP1B in Reelin-dependent Neuronal Migration. Cereb Cortex. 15:11341145.

Hochgerner H, Zeisel A, Lönnerberg P, Linnarsson S. 2018. Conserved properties of 647 dentate gyrus neurogenesis across postnatal development revealed by single-cell RNA sequencing. Nat Neurosci. 21:290-299.

Hussman JP, Chung RH, Griswold AJ, Jaworski JM, Salyakina D, Ma D, Konidari I, Whitehead PL, Vance JM, Martin ER, Cuccaro ML, Gilbert JR, Haines JL, PericakVance MA. 2011. A noise-reduction GWAS analysis implicates altered regulation of neurite outgrowth and guidance in autism. Mol Autism. 2.

JP H, RH C, AJ G, JM J, D S, D M, I K, PL W, JM V, ER M, ML C, JR G, JL H, MA P-V. 2011. A noise-reduction GWAS analysis implicates altered regulation of neurite outgrowth and guidance in autism. Mol Autism. 2.

Karpowicz P, Willaime-Morawek S, Balenci L, Deveale B, Inoue T, Van Der Kooy D. 2009. E-Cadherin regulates neural stem cell self-renewal. J Neurosci. 29:3885-3896.

Kawauchi T, Hoshino M. 2008. Molecular Pathways Regulating Cytoskeletal Organization and Morphological Changes in Migrating Neurons. Dev Neurosci. 30:36-46.

Kempermann G, Song H, Gage FH. n.d. Neurogenesis in the Adult Hippocampus.

663 Kiselyov V V., Skladchikova G, Hinsby AM, Jensen PH, Kulahin N, Soroka V, Pedersen N, Tsetlin V, Poulsen FM, Berezin V, Bock E. 2003. Structural basis for a direct interaction between FGFR1 and NCAM and evidence for a regulatory role of ATP. Structure. 11:691-701. 
Kulahin N, Walmod PS. 2010. The neural cell adhesion molecule NCAM2/OCAM/RNCAM, a close relative to NCAM. Adv Exp Med Biol. 663:403-420.

Kumar A, Pareek V, Faiq MA, Ghosh SK, Kumari C. 2019. ADULT NEUROGENESIS IN HUMANS: A Review of Basic Concepts, History, Current Research, and Clinical Implications. Innov Clin Neurosci. 16:30.

Leshchyns'Ka I, Liew HT, Shepherd C, Halliday GM, Stevens CH, Ke YD, Ittner LM, Sytnyk V. 2015. Aß-dependent reduction of NCAM2-mediated synaptic adhesion contributes to synapse loss in Alzheimer's disease. Nat Commun. 6:8836.

Llorens-Bobadilla E, Zhao S, Baser A, Saiz-Castro G, Zwadlo K, Martin-Villalba A. 2015. Single-Cell Transcriptomics Reveals a Population of Dormant Neural Stem Cells that Become Activated upon Brain Injury. Cell Stem Cell. 17:329-340.

Makino T, McLysaght A. 2010. Ohnologs in the human genome are dosage balanced and frequently associated with disease. Proc Natl Acad Sci U S A. 107:9270-9274.

Marthiens V, Kazanis I, Moss L, Long K, Ffrench-Constant C. 2010. Adhesion molecules in the stem cell niche - More than just staying in shape? J Cell Sci.

Morante-Redolat JM, Porlan E. 2019. Neural stem cell regulation by adhesion molecules within the subependymal niche. Front Cell Dev Biol.

Morizur L, Chicheportiche A, Gauthier LR, Daynac M, Boussin FD, Mouthon MA. 2018. Distinct Molecular Signatures of Quiescent and Activated Adult Neural Stem Cells

N U, F G. 2014. Neurogenesis in the embryonic and adult brain: same regulators, different roles. Front Cell Neurosci. 8.

Paoloni-Giacobino A, Chen H, Antonarakis SE. 1997. Cloning of a novel human neural cell

Parcerisas A, Ortega-Gascó A, Pujadas L, Soriano E. 2021. The Hidden Side of NCAM 
reveal functions of NCAM2 in neural cytoskeleton organization. Int J Mol Sci. 22.

Parcerisas A, Pujadas L, Ortega-Gascó A, Perelló-Amorós B, Viais R, Hino K, Figueiro-Silva Dendritic and Axonal Differentiation through the Cytoskeletal Proteins MAP2 and 14-3-3. Cereb Cortex. 30:3781-3799.

Parr JR, Lamb JA, Bailey AJ, Monaco AP. 2006. Response to paper by Molloy et al.: Linkage on 21q and 7q in autism subset with regression [2]. Mol Psychiatry.

Pébusque MJ, Coulier F, Birnbaum D, Pontarotti P. 1998. Ancient large-scale genome duplications: Phylogenetic and linkage analyses shed light on chordate genome evolution. Mol Biol Evol. 15:1145-1159.

Petit F, Plessis G, Decamp M, Cuisset JM, Blyth M, Pendlebury M, Andrieux J. 2015. 21q21 deletion involving NCAM2: Report of 3 cases with neurodevelopmental disorders. Eur J Med Genet. 58:44-46.

Povlsen GK, Berezin V, Bock E. 2008. Neural cell adhesion molecule-180-mediated homophilic binding induces epidermal growth factor receptor (EGFR) down-

Scholz C, Steinemann D, Mälzer M, Roy M, Arslan-Kirchner M, Illig T, Schmidtke J, regulation and uncouples the inhibitory function of EGFR in neurite outgrowth. J

Seri B, García-Verdugo JM, Collado-Morente L, McEwen BS, Alvarez-Buylla A. 2004. Cell Neurochem. 104:624-639.

Rasmussen KK, Falkesgaard MH, Winther M, Roed NK, Quistgaard CL, Teisen MN, Edslev SM, Petersen DL, Aljubouri A, Christensen C, Thulstrup PW, Lo Leggio L, Teilum K,

Sheng L, Leshchyns'Ka I, Sytnyk V. 2015. Neural cell adhesion molecule 2 promotes the formation of filopodia and neurite branching by inducing submembrane increases in Ca2+ levels. J Neurosci. 35:1739-1752. 
Cascades underlying Adult Neurogenesis. Cell Stem Cell. 17:360-372.

Shin J, Berg DA, Zhu Y, Shin JY, Song J, Bonaguidi MA, Enikolopov G, Nauen DW, Christian KM, Ming GL, Song H. 2015. Table S6. Single-cell gene expression table according to the pseudotime progression. In: hin J, Berg DA, Zhu Y, Shin JY, Song J, Bonaguidi MA, Enikolopov G, Nauen DW, Christian KM, Ming GL, Song H. Single-Cell RNA-Seq with Waterfall Reveals Molecular Cascades underlying Adult Neurogenesis. Cell Stem Cell. 17:360-372.

Simó S, Jossin Y, Cooper JA. 2010. Cullin 5 regulates cortical layering by modulating the speed and duration of Dab1-dependent neuronal migration. J Neurosci. 30:56685676.

Teixeira CM, Kron MM, Masachs N, Zhang H, Lagace DC, Martinez A, Reillo I, Duan X, Bosch C, Pujadas L, Brunso L, Song H, Eisch AJ, Borrell V, Howell BW, Parent JM, Soriano E. 2012. Cell-autonomous inactivation of the reelin pathway impairs adult neurogenesis in the hippocampus. J Neurosci. 32:12051-12065.

Walker TL, Kempermann G. 2014. One mouse, two cultures: isolation and culture of adult neural stem cells from the two neurogenic zones of individual mice. J Vis Exp. e51225.

Wang YAZ, Plane JM, Jiang P, Zhou CJ, Deng W. 2011. Concise review: Quiescent and

Winther M, Berezin V, Walmod PS. 2012. NCAM2/OCAM/RNCAM: Cell adhesion molecule with a role in neuronal compartmentalization. Int J Biochem Cell Biol.

Xie XP, Laks DR, Sun D, Poran A, Laughney AM, Wang Z, Sam J, Belenguer G, Fariñas I, Elemento O, Zhou X, Parada LF. 2020. High-resolution mouse subventricular zone stem-cell niche transcriptome reveals features of lineage, anatomy, and aging. Proc 
Natl Acad Sci U S A. 117:31448-31458.

766 Yao B, Christian KM, He C, Jin P, Ming GL, Song H. 2016. Epigenetic mechanisms in 767 neurogenesis. Nat Rev Neurosci.

768 Zhang L, Zhang X. 2018. Factors Regulating Neurogenesis in the Adult Dentate Gyrus. In:

769 The Hippocampus - Plasticity and Functions. InTech.

770 Zhao C, Deng W, Gage FH. 2008. Mechanisms and Functional Implications of Adult 771 Neurogenesis. Cell.

772 
774 Figure 1. Expression pattern of NCAM2 in the hippocampus progenitor cells.

775 A) Immunohistochemical characteritzation of NCAM2 expression in GFAP/Sox2 776 progenitor cells in P45 mice hippocampus. Arrowheads label NCAM2/GFAP/Sox2 777 positive cells. B) NCAM2 expression in Nestin positive cells in the subgranular zone of 778 P45 mice. Arrowheads label NCAM2/Nestin cells. C) Double immunostaining of NCAM2 779 and Tbr2 at P45. Arrowheads label Tbr2 positive cells that present low NCAM2 signal. 780 ML: molecular layer; GL: granule layer; H: hilus. Scale bar: A) $50 \mu \mathrm{m}, \mathrm{B}, \mathrm{C}) 20 \mu \mathrm{m}$.

781 Figure 2. NCAM2 overexpression modulates adult neurogenesis in the hippocampus.

782 A) Representative images of GFP positive cells from the dentate gyrus of mice injected 783 with control, ShNCAM2, or NCAM2 overexpressing viruses (NCAM2.1 and NCAM2.2) at 7844 weeks after injection. Control and ShNCAM2 positive cells show a granule cell morphology while RGP-like phenotype was observed in many cells infected with NCAM2.1 or NCAM2.2 overexpressing viruses. Scale bar: $50 \mu \mathrm{m}$. B) GFP immunogold electron microscopy images of animals infected with control, NCAM2.1 OE or NCAM2.2 OE viruses and sacrificed 4 weeks post-surgery. Control images show densely GFPlabelled granule cells and RGPs. In NCAM2.1 and NCAM2.2 OE mice, the number of labelled granule cells is dramatically decreased. Nevertheless, RGPs located in the SGZ still appear labelled with GFP. GC: granule cell; RGP: radial glia progenitor. Scale bar: 2 $\mu \mathrm{m}$.

Figure 4. Immunohistochemical characterization of NCAM2 overexpressing progenitor cells.

A) Immunostaining of GFP positive cells with GFAP and Sox2 RGPs markers from animals sacrificed 1 week post-injection. B) Immunostaining of GFP positive cells with GFAP and Sox2 RGPs markers from animals sacrificed 4 weeks post-injection. C-D) Time course quantification of the GFP/Sox2/GFAP positive cells in mice injected with control, NCAM2.1 OE or NCAM2.2 OE viruses at 3 days, 1 week, 2 weeks and 4 weeks post-

800 injection. $\mathrm{N}=2-3$ animals, 5-10 slices per animal. Data are presented as mean $\pm \mathrm{SEM}$; dots 801 represent average values for individual animals (5-10 slices per animal, 20-50 cells per 802 animal); $\mathrm{N}=2-3$ animals per group at 3 days (control) 1, 2 and 4 weeks post-injection; 
ANOVA, Tukey's comparison post-hoc test; * $\mathrm{P}<0.05, * * \mathrm{P}<0.01, * * * \mathrm{P}<0.001$,

$\mathrm{P}<0.0001$. In light gray bars, representation of NCAM2.1 and NCAM2.2 groups at 3 days post-injection ( $\mathrm{N}=1$ animals per group, qualitative study excluded form statistical analysis). In Fig. 4D, gray * differences between Control and NCAM2.1; black * differences between Control and NCAM2.2; • differences between NCAM2.1 and NCAM2.2. Arrowheads label GFP/Sox2/GFAP positive GFP-cells. ML: molecular layer; GL: granule layer; H: hilus. Scale bar: A, B) $20 \mu \mathrm{m}$.

Figure 5. Immunohistochemical characterization of NCAM2 overexpressing neurons.

811 A) Immunostaining of GFP positive cells with DCX as a markers for neuroblasts (type III

812 progenitors) and immature neurons from animals sacrificed 4 week post-injection. B)

813 Immunostaining of GFP positive cells with NeuN as a markers for mature neurons from

814 animals sacrificed 4 week post-injection. C) Quantifications of GFP/DCX positive cells in

815 mice injected with control, NCAM2.1 OE or NCAM2.2 OE viruses at 2 and 4 weeks post-

816 injection. $\mathrm{N}=2-4$ animals per group, $5-6$ slices ( $>50$ cells per animal in the controls; $15-30$

817 cells per animal in the NCAM2 OE conditions). D) Quantification of GFP/NeuN positive

818 cells in animals injected with control, NCAM2.1 OE or NCAM2.2 OE 4 weeks after

819 transduction. $\mathrm{N}=4$ animals per group, 5 slices per animal ( $>50$ cells per animal). Data are

820 presented as mean \pm SEM; differences between experimental groups ANOVA, Tukey's

821 comparison post-hoc test; ${ }^{* *} \mathrm{P}<0.01$; differences between time points Student's t-test;

$822 * \mathrm{P}<0.05$. Arrowheads label DCX or NeuN positive GFP-cells; arrows label NeuN negative

823 GFP-cells. ML: molecular layer; GL: granule layer; H: hilus. Scale bar: A, B) $20 \mu \mathrm{m}$.

824 Figure 6. NCAM2 overexpression do not arrest embrionary RGPs but affects neuronal 825 migration.

826 A) Representative images from the reporter gene GFP in electroporated neurons in 827 cortical sections from P0 mice. E15-born neurons were electroporated with control (left 828 panel) and overexpression vectors. Sections were counterstained with DAPI. B)

829 Distribution of transfected cells within cortical layers was quantified at PO by dividing 830 cortical thickness in 10 BINs. Data are presented as the ratio of neurons with somas 831 located in each BIN. Overexpression of NCAM2.2 isoform or simultaneous expression of 832 both isoforms (NCAM2.1+NCAM2.2) induce a reduced proportion of cells in the upper 833 BIN. $\mathrm{N}=5-8$ animals electroporated with control or overexpression constructs; *** 
$834 \mathrm{P}<0.001$; two-way ANOVA, Bonferroni comparison post hoc test. C) Representative

835 images from the reporter gene GFP in electroporated neurons in cortical sections from

836 P5 mice. E15-born neurons were electroporated with control (left panel) and

837 overexpression vectors for both isoforms (NCAM2.1+NCAM2.2; right panel). D)

838 Distribution of transfected cells within cortical layers was quantified at P5 in 10 BINs.

839 Data are presented as the ratio of neurons with somas located in each BIN. No

840 differences were found within neuronal distribution between control and NCAM2-

841 overexpressing neurons. $\mathrm{N}=6$ electroporated animals with the constructs; two-ways

842 ANOVA, Bonferroni comparison post hoc test. E) Higher magnification of representative

843 images from transfected neurons at P0. Neurons show normal pyramidal neuronal

844 morphology. CP, cortical plate; IZ, intermediate zone; MZ, marginal zone; SVZ,

845 subventricular zone; I-VI, cortical layers. Scale bars: A,D) $50 \mu \mathrm{m}$; E) $10 \mu \mathrm{m}$.

846 Figure 7. NCAM2 expression levels affect the proliferation of NSCs grown as 847 neurospheres.

848 A) Scheme showing the protocol for the obtention of post-natal mouse neurospheres

849 from the neurogenic niches. Progenitor cells were isolated and grown as neurospheres

850 A.1) Neurospheres where infected with control, NCASM2 overexpressing or ShNCAM2

851 viruses, selected by flow cytometry and plated in non-adherent plates. The area of the

852 infected neurospheres was analysed by Scan-R microscopy for 5 consecutive days. A.2)

853 Cells were plated in adherent coverslips, infected with control, NCAM2 overexpressing

854 or ShNCAM2 viruses and maintained 5 days in differentiation conditions before fixation.

855 B) Representative images of control, ShNCAM2, or NCAM2 overexpressing 856 neurospheres after 3 days in vitro. C) Quantification of the time-course progress for the

857 area of neurospheres for 5 consecutive days after sorting of infected cells. $\mathrm{N}=100-300$

858 neurospheres per condition, 1 independent experiment. D) Comparison of the area of 859 neurospheres at 3 days in vitro. E) Histograms of control, NCAM2.1 OE, NCAM2.2 OE and

860 ShNCAM2 neurospheres distribution according to their area 3 days after FACS selection.

861 Coloured bars label percentile 50. Data are presented as mean \pm SEM; Kruskal-Wallis 862 test, $* * * \mathrm{P}<0.001$. Scale bar: B) $100 \mu \mathrm{m}$. 


\section{Figure 8. Model of RGPs regulation by NCAM2 expression levels in the hippocampus.}

864 Schematic representation of the proposed model for NSC regulation by NCAM2

865 expression. RGPs (Type I cells) are GFAP/Sox2/Nestin positive cells and are maintained

866 in a quiescent state in the SGZ of the dentate gyrus. Upon activation, they generate Tbr2

867 positive proliferating intermediate progenitors (Type 2 cells). Those transit-amplifying

868 progenitors produce neuroblasts (Type 3 cells) that express DCX and differentiate into

869 NeuN positive granule cells. New-born neurons mature and become functional neurons

870 of the hippocampal circuits. This process is regulated by different intrinsic and extrinsic

871 factors, such as growth factors. We postulate that the levels of cell adhesion molecules

872 such as NCAM2 protein are crucial for the regulation of NSC quiescence, the activation

873 of proliferation and for the proper neuronal differentiation and maturation in later

874 stages (Shin et al. 2015; Morizur et al. 2018; Parcerisas et al. 2020). 

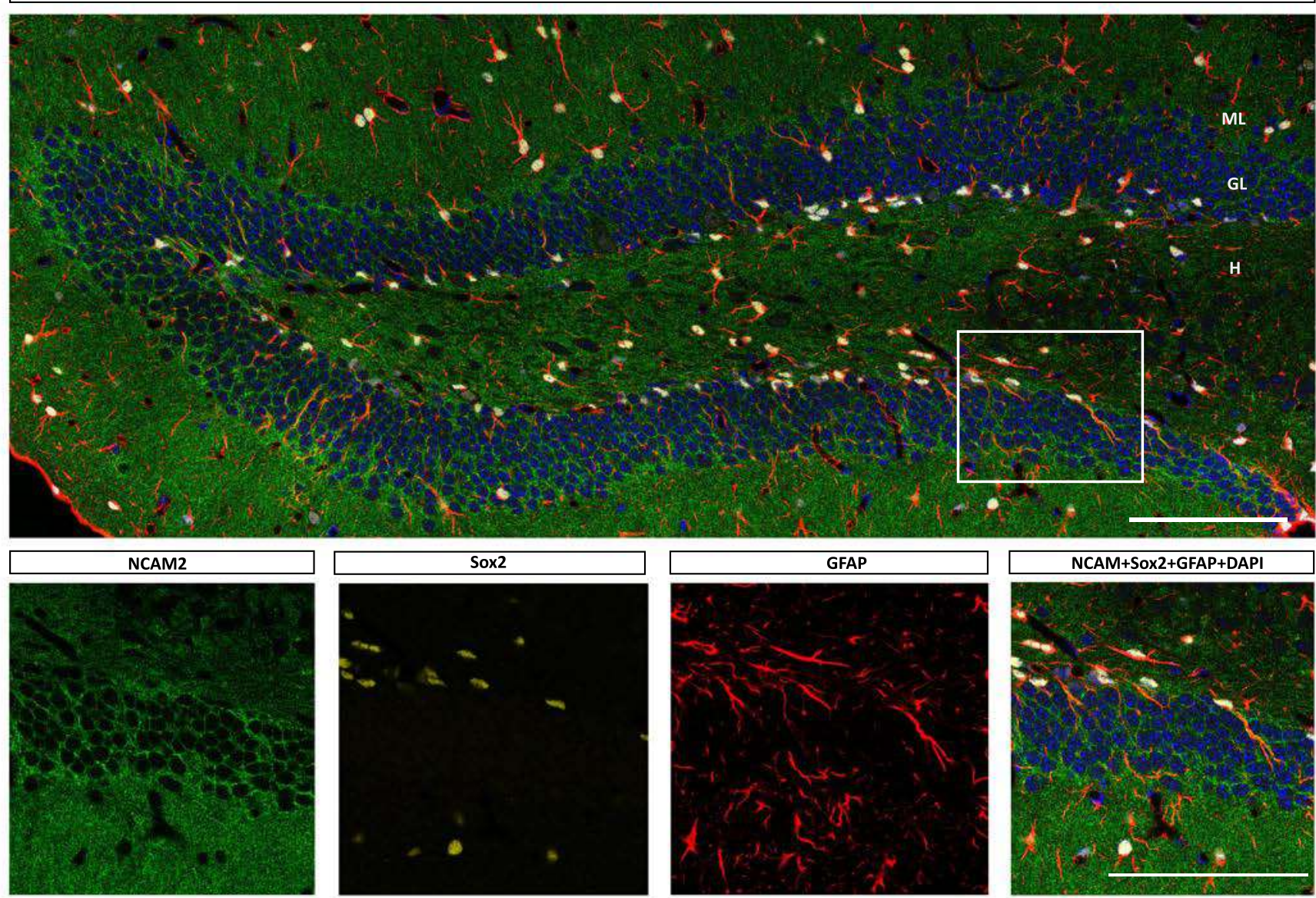

B

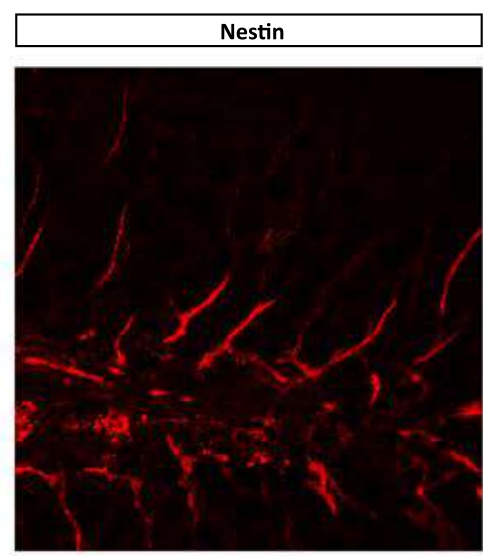

Tbr2

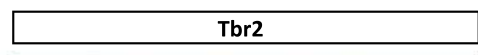

NCAM2+Nestin+DAPI

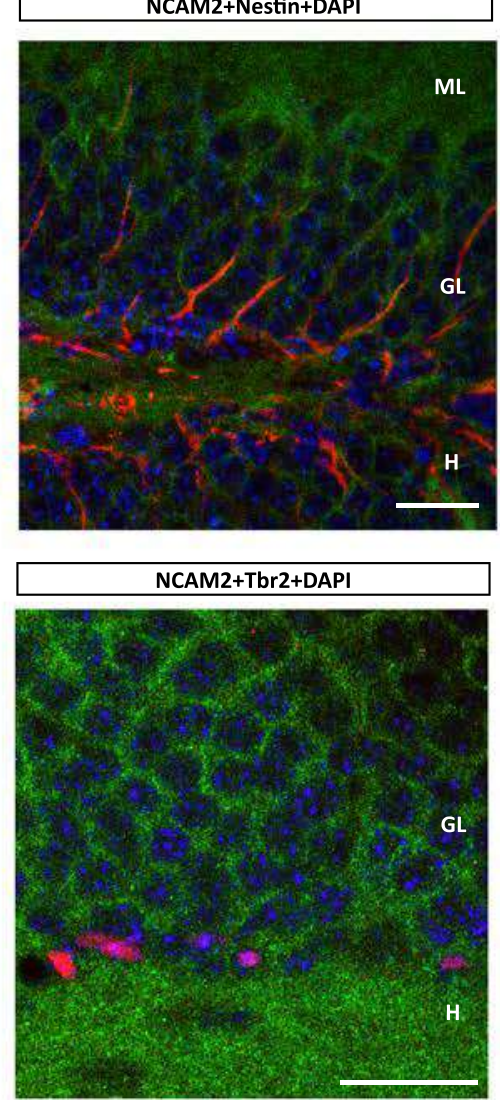


A
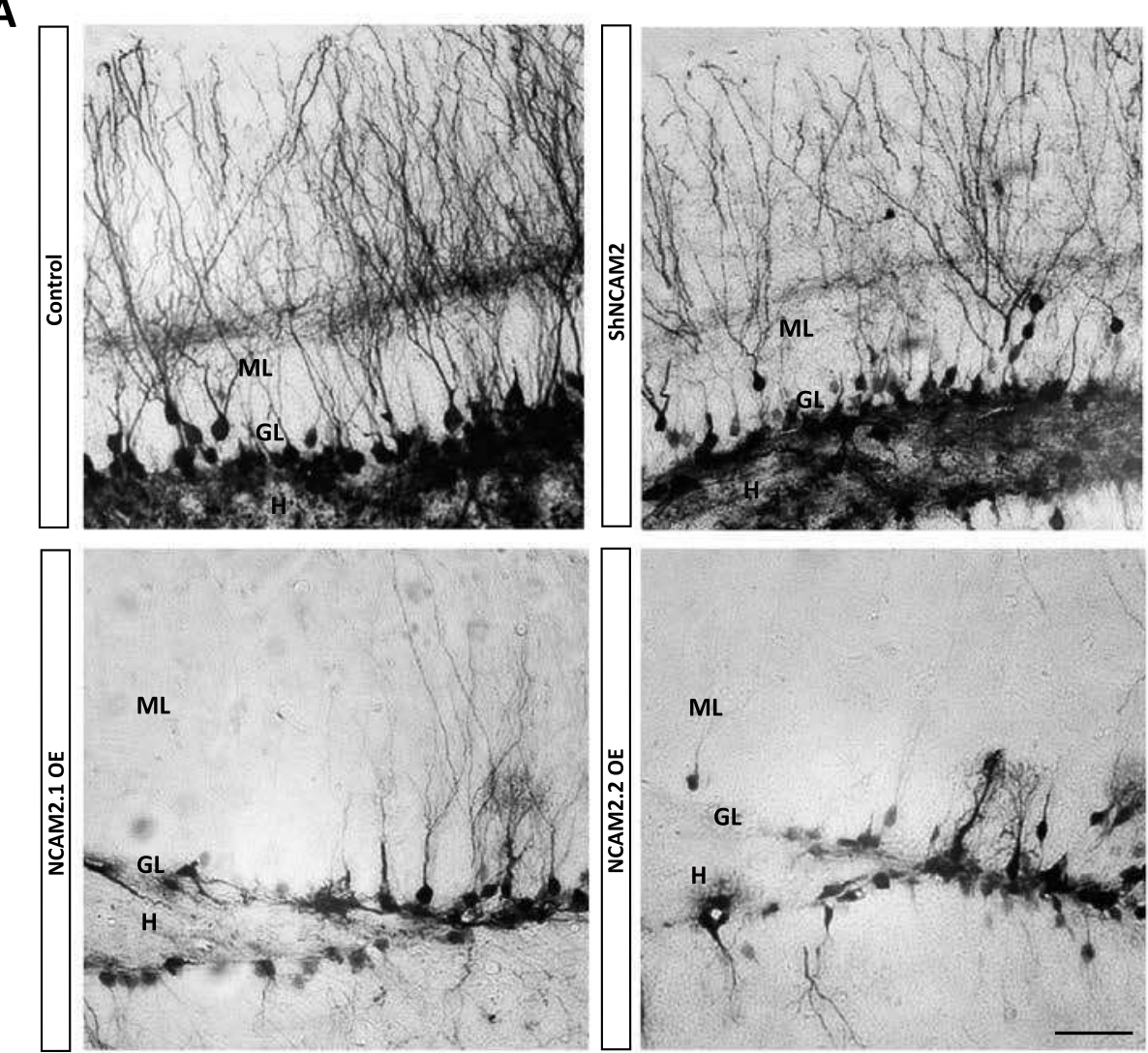

B
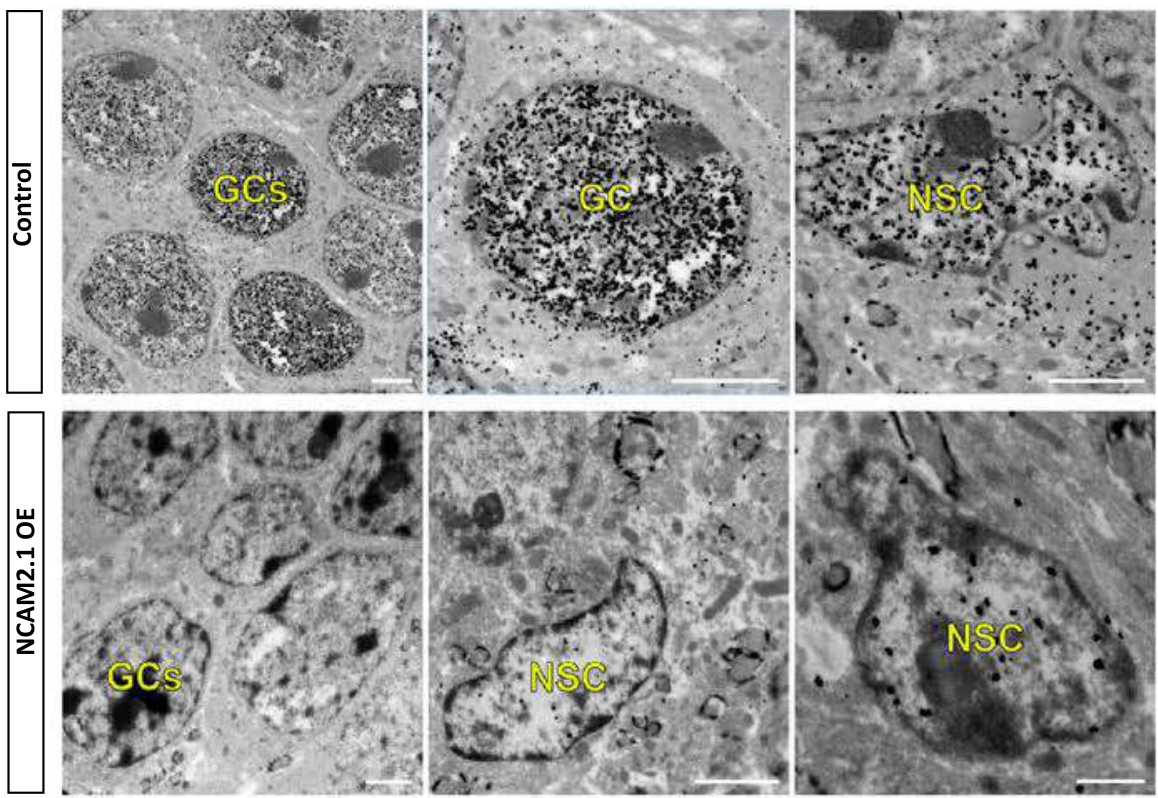

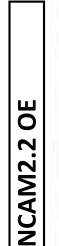
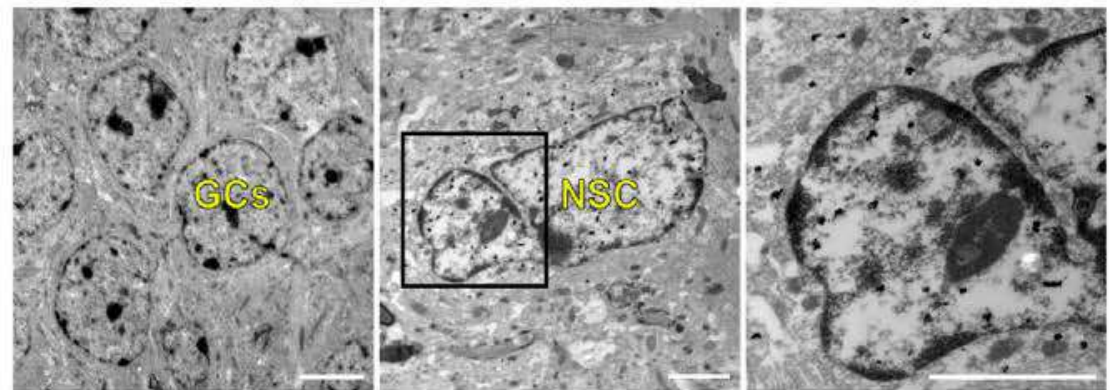

Figure 2 
A

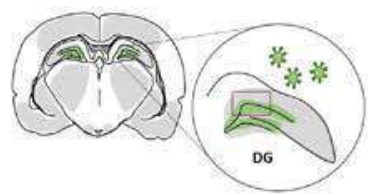

Virus injection

B

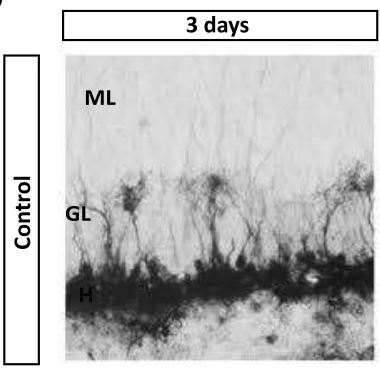

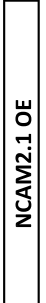

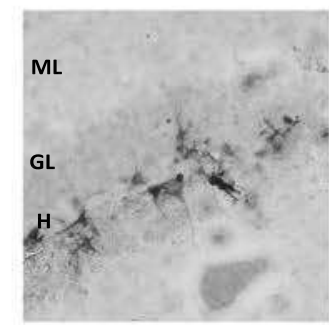

แu

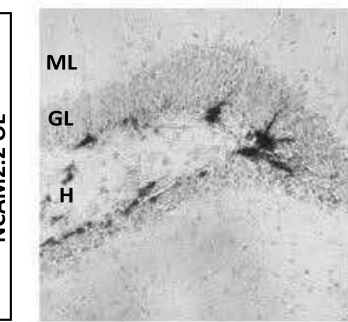

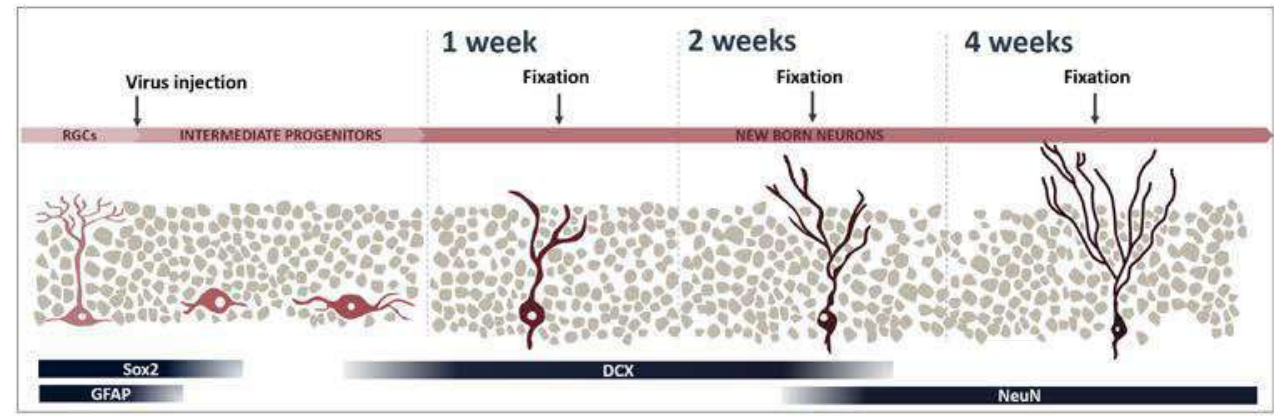
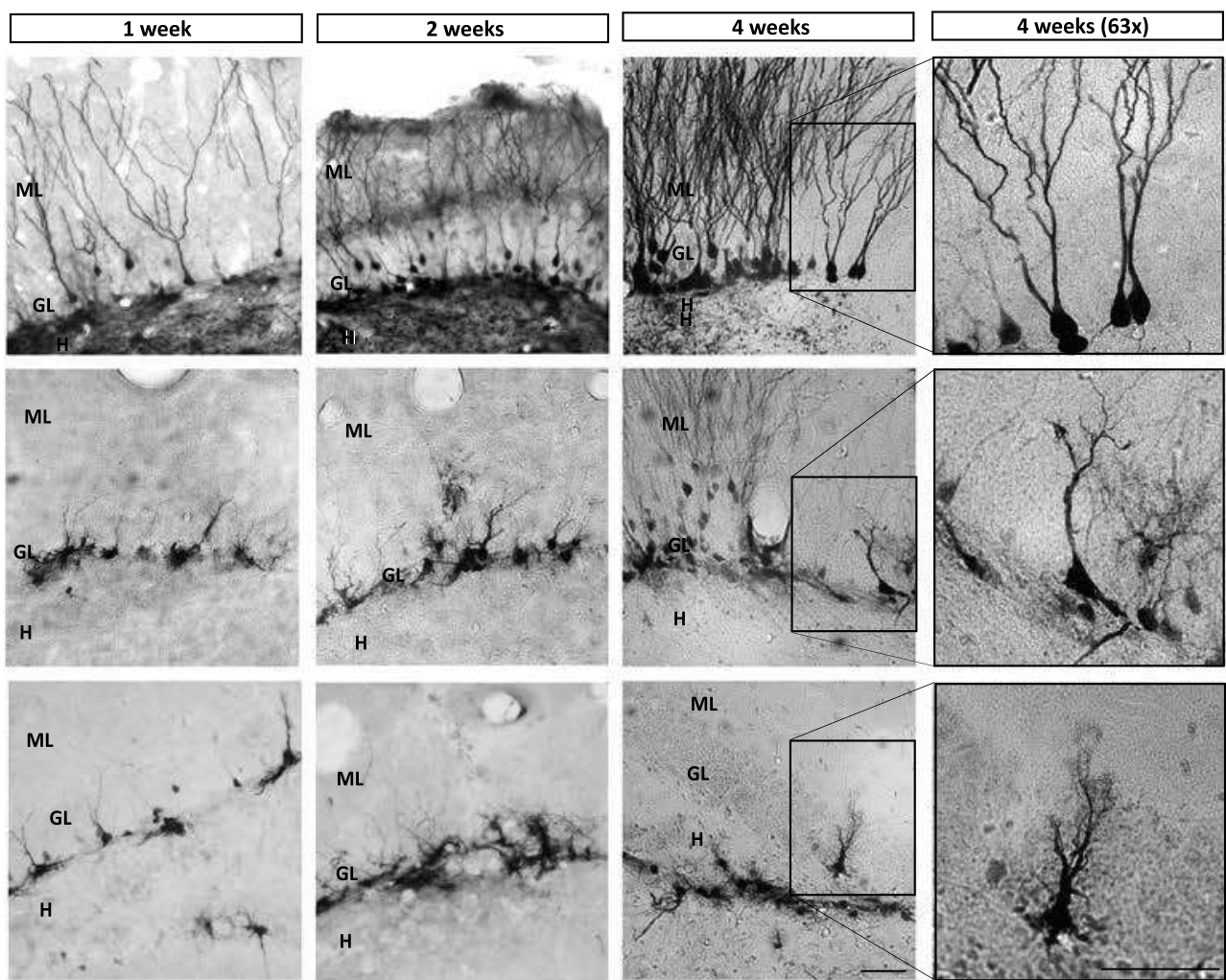

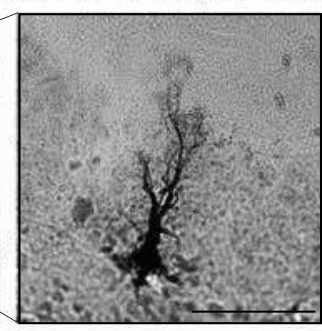

Figure 3 
bioRxiv preprint doi: https://doi.org/10.1101/2022.02.03.478938; this version posted February 3, 2022. The copyright holder for this preprint (which was not certified by peer review) is the author/funder. All rights reserved. No reuse allowed without permission.

A

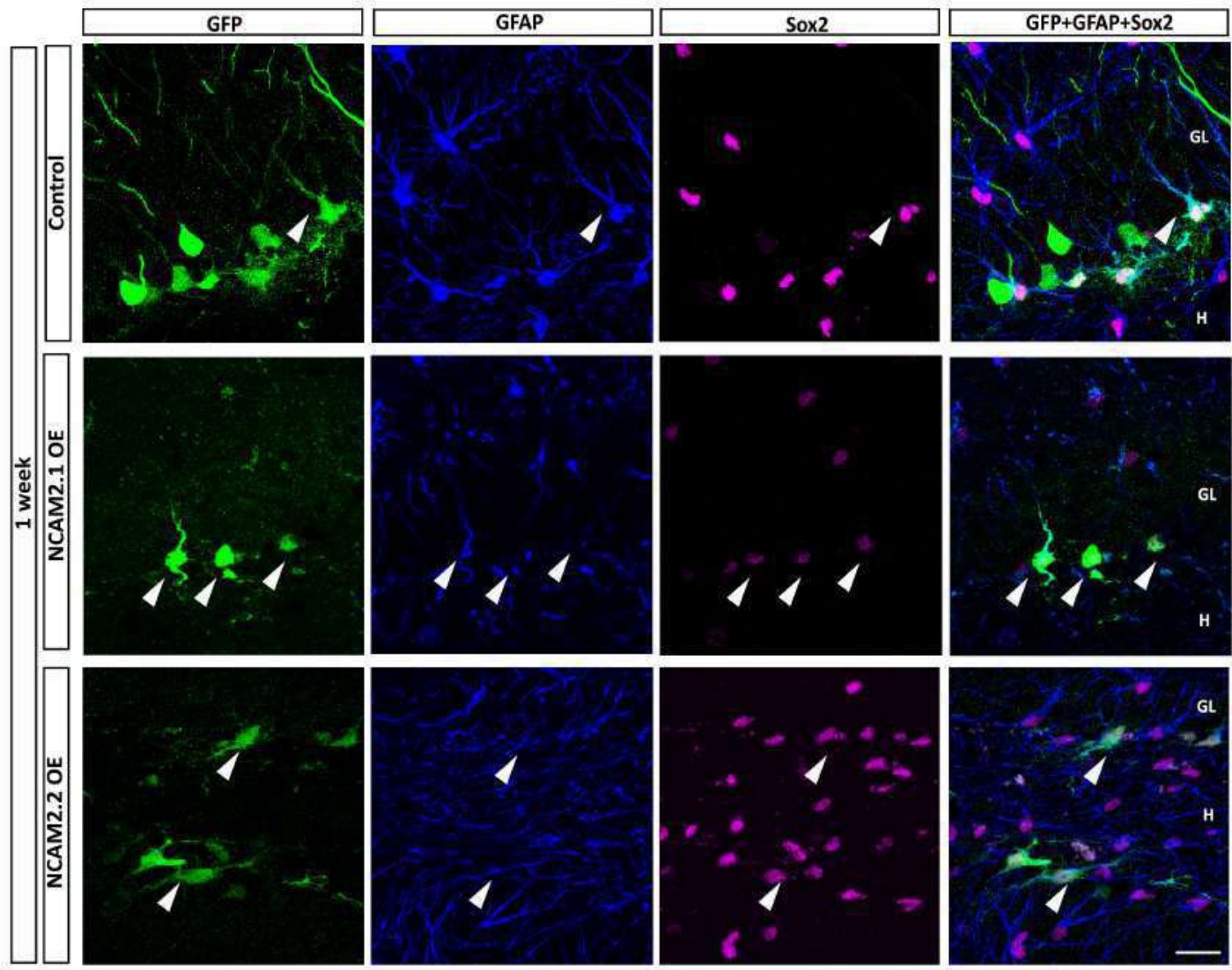

B
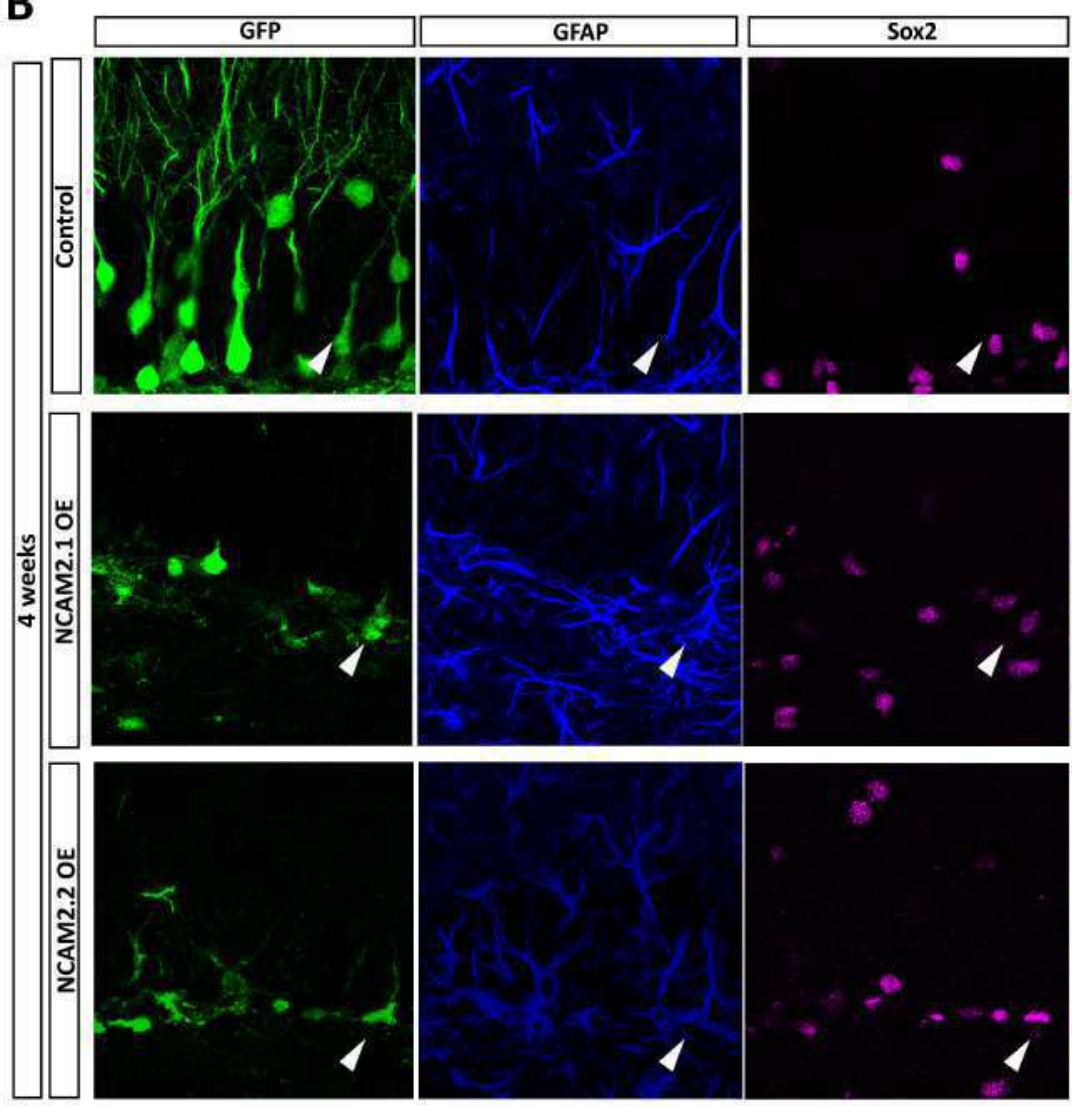
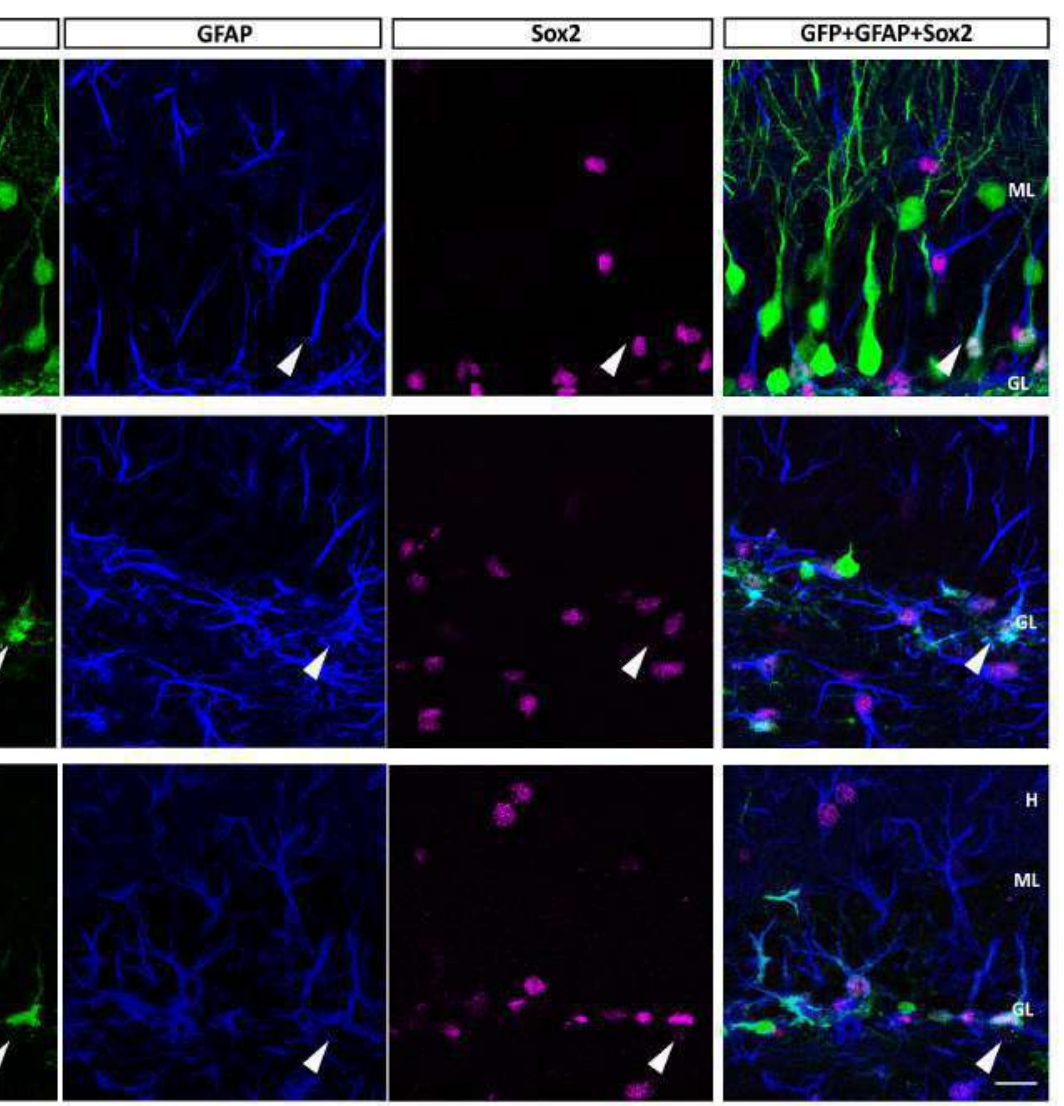

C

GFP/GFAP/Sox2 positive cells
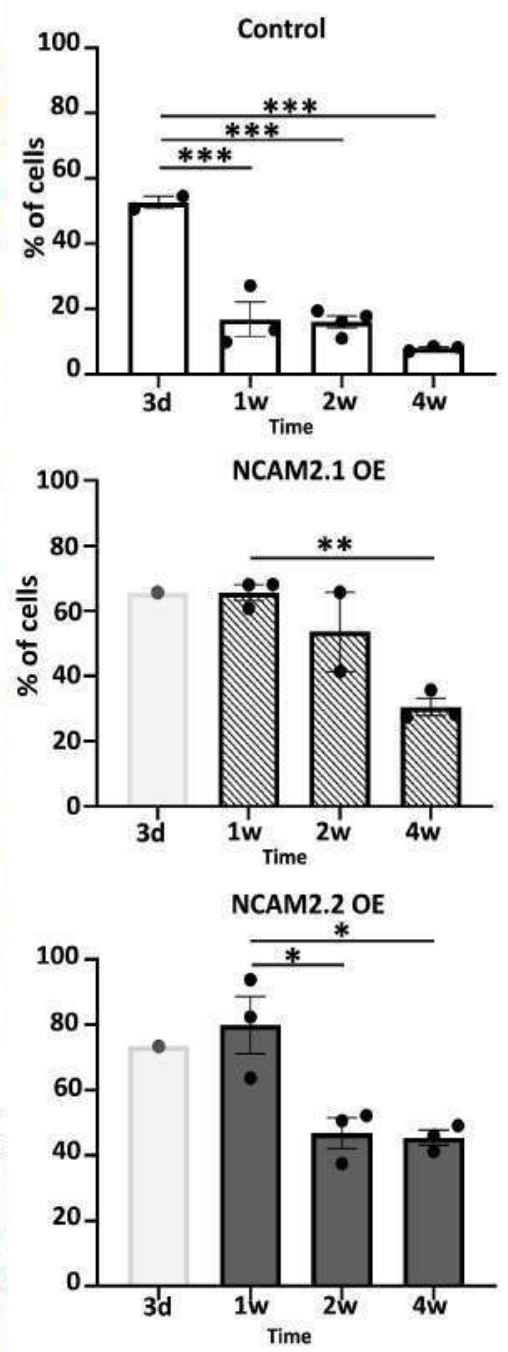

D

GFP/GFAP/Sox2 cells progression

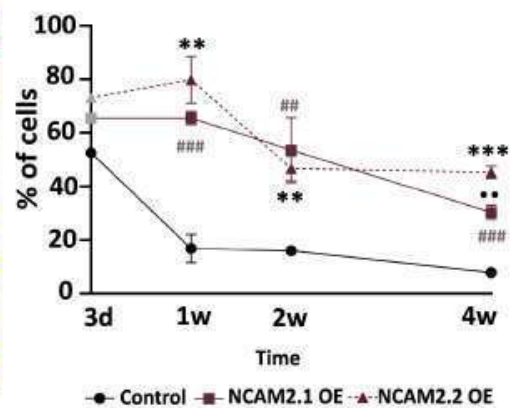


A

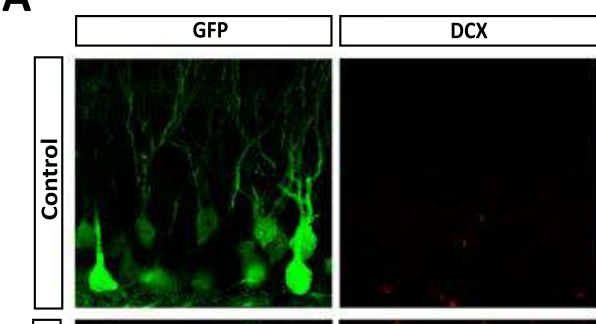

ט.

ప્'

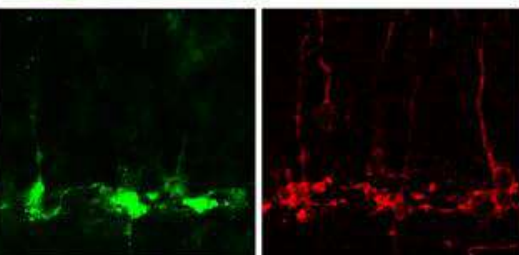

"u

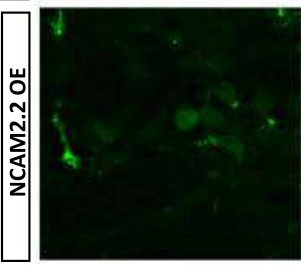

C

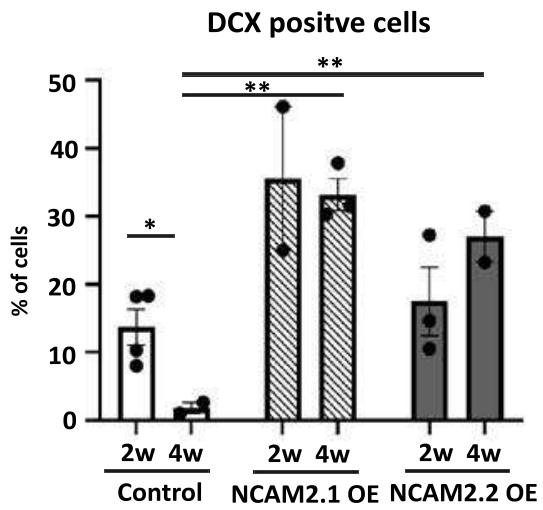

B
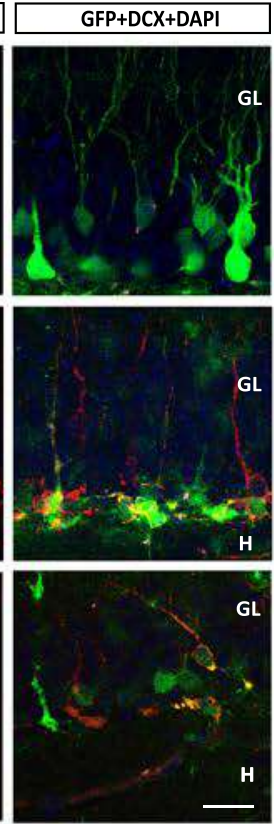

H
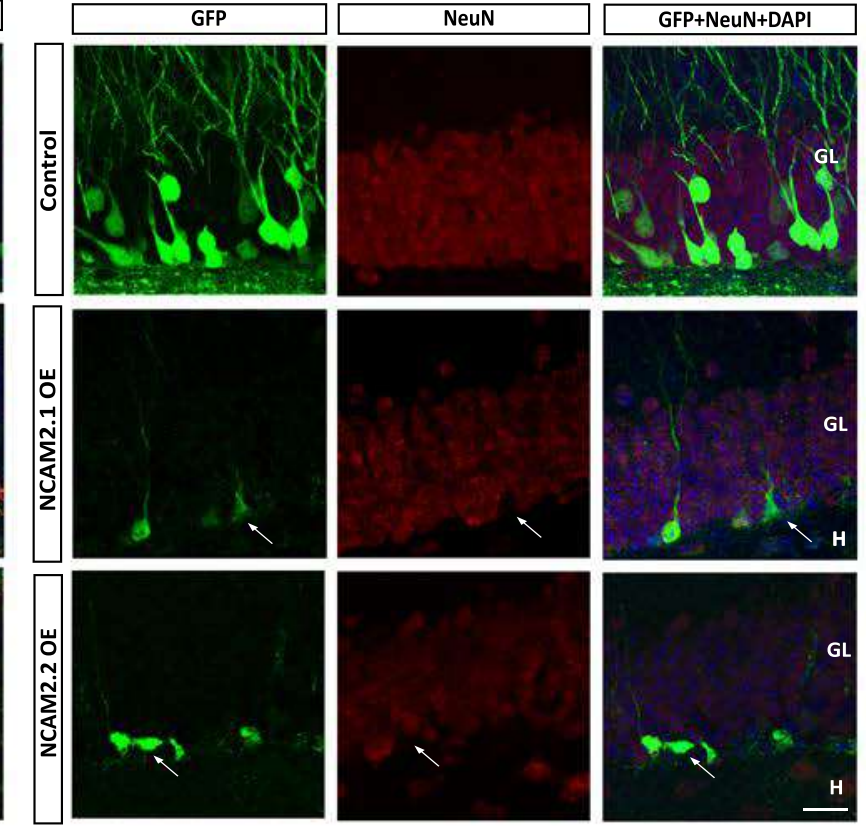

D

GFP/NeuN positve cells $4 w$

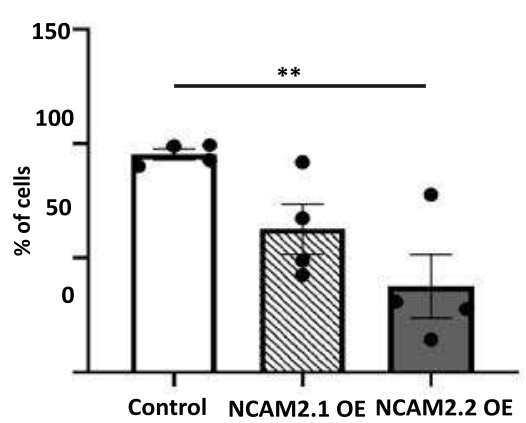

Figure 5 
A
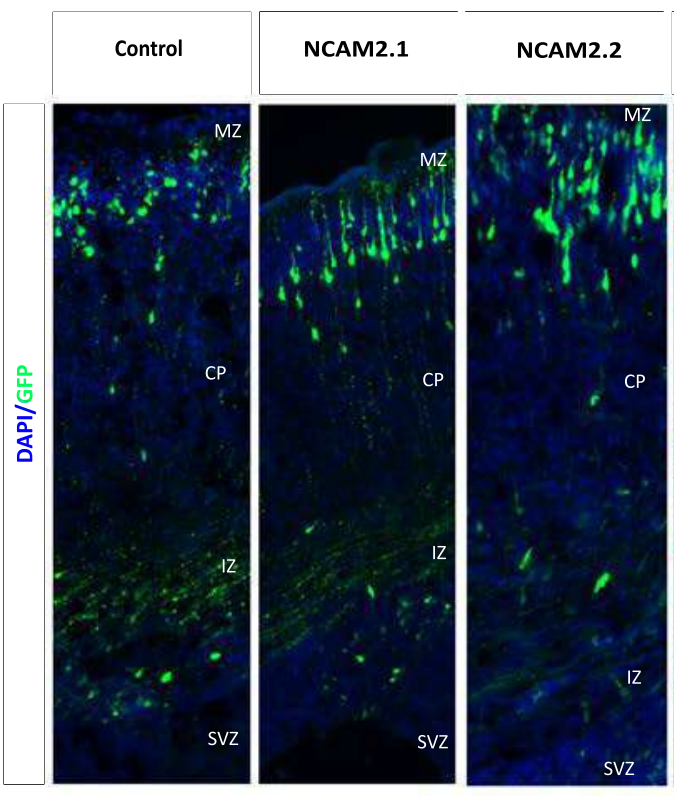

\section{NCAM2.1 \\ NCAM2.2}

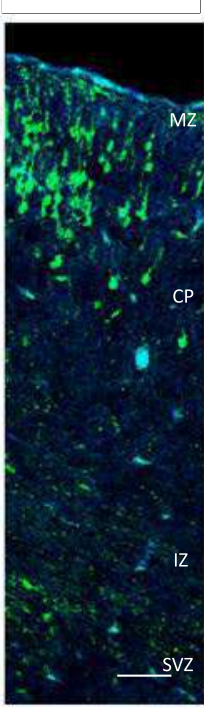

D

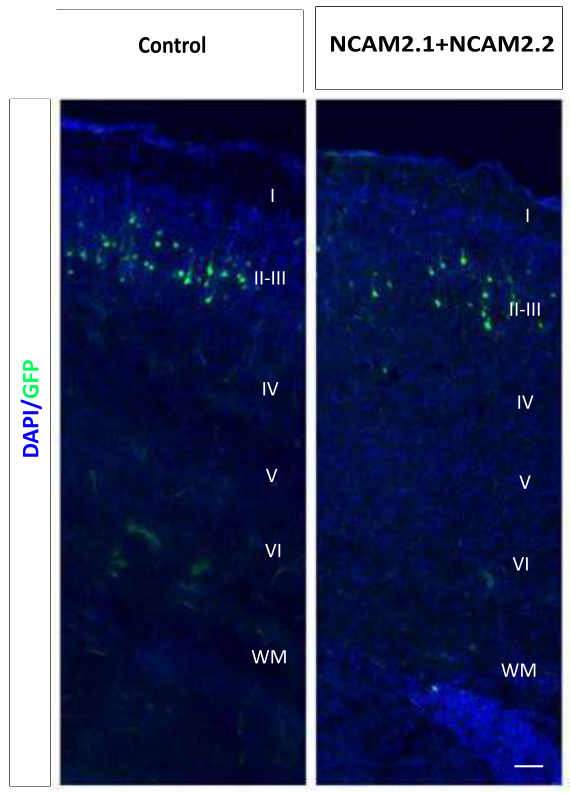

Neuronal
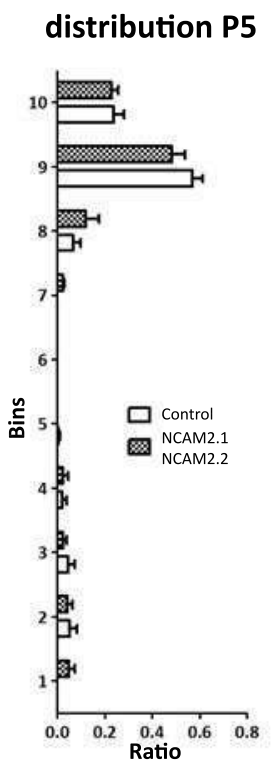

B
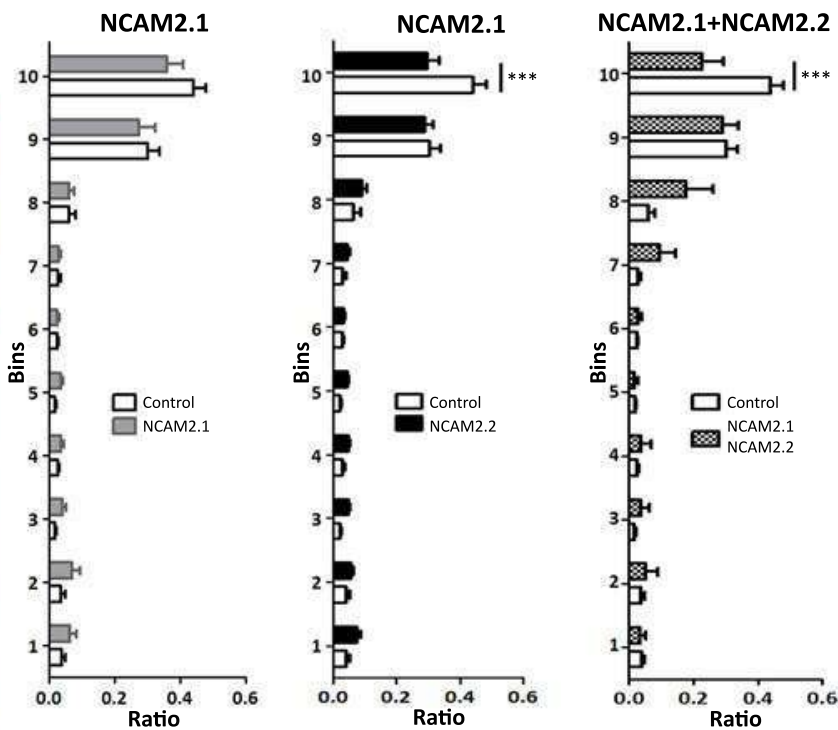

E

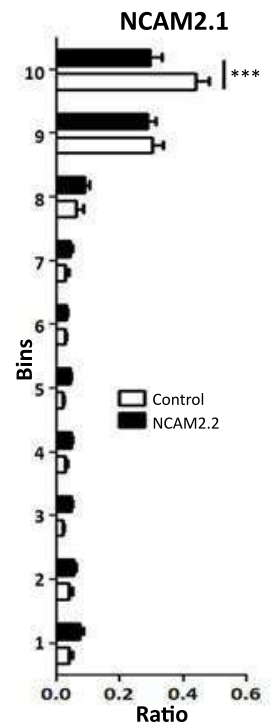

\section{Neuronal distribution PO}

Neuronal phenotype

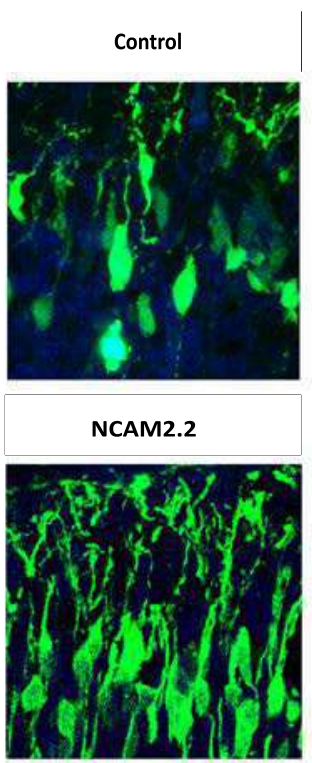

NCAM2.1

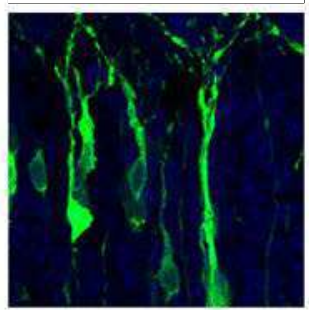

NCAM2.1+NCAM2.2

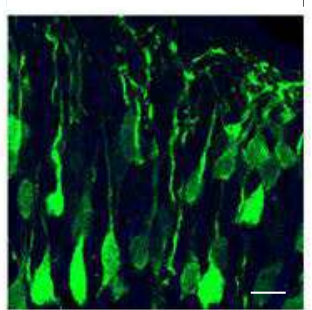

Figure 6 
A

NSCS CULTURE

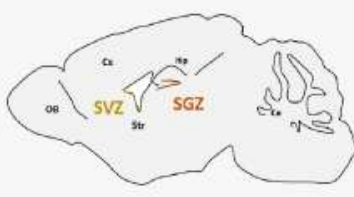

NSCs isolation from the $5 \mathrm{SZ} \mathrm{P7} / \mathrm{P8}$ mice

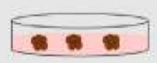

Neurospheres subculture

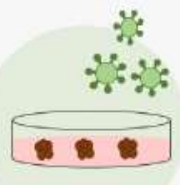

Infection

B

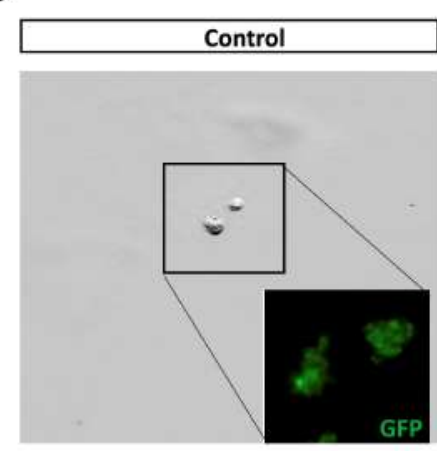

C

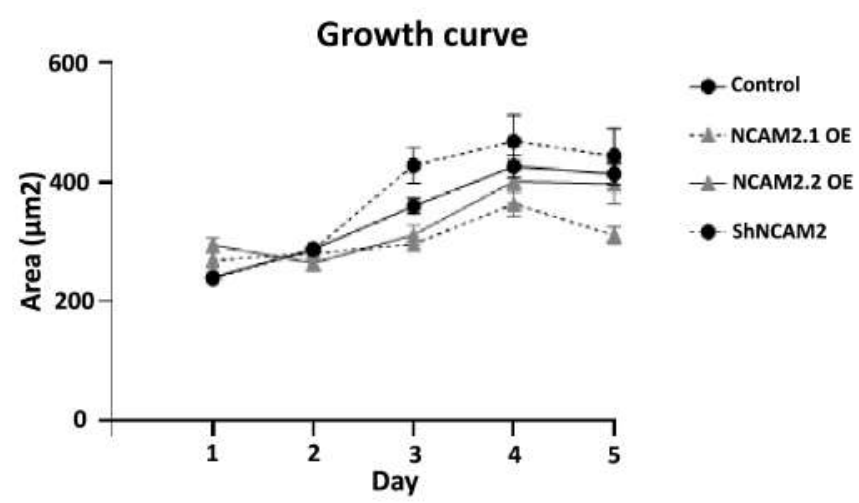

D

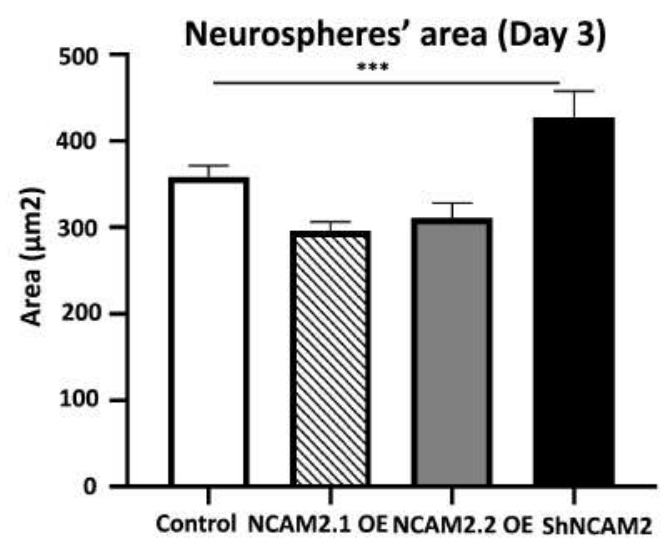

NCAM2.1 OE

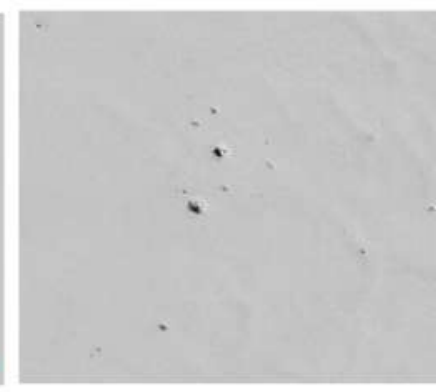

E
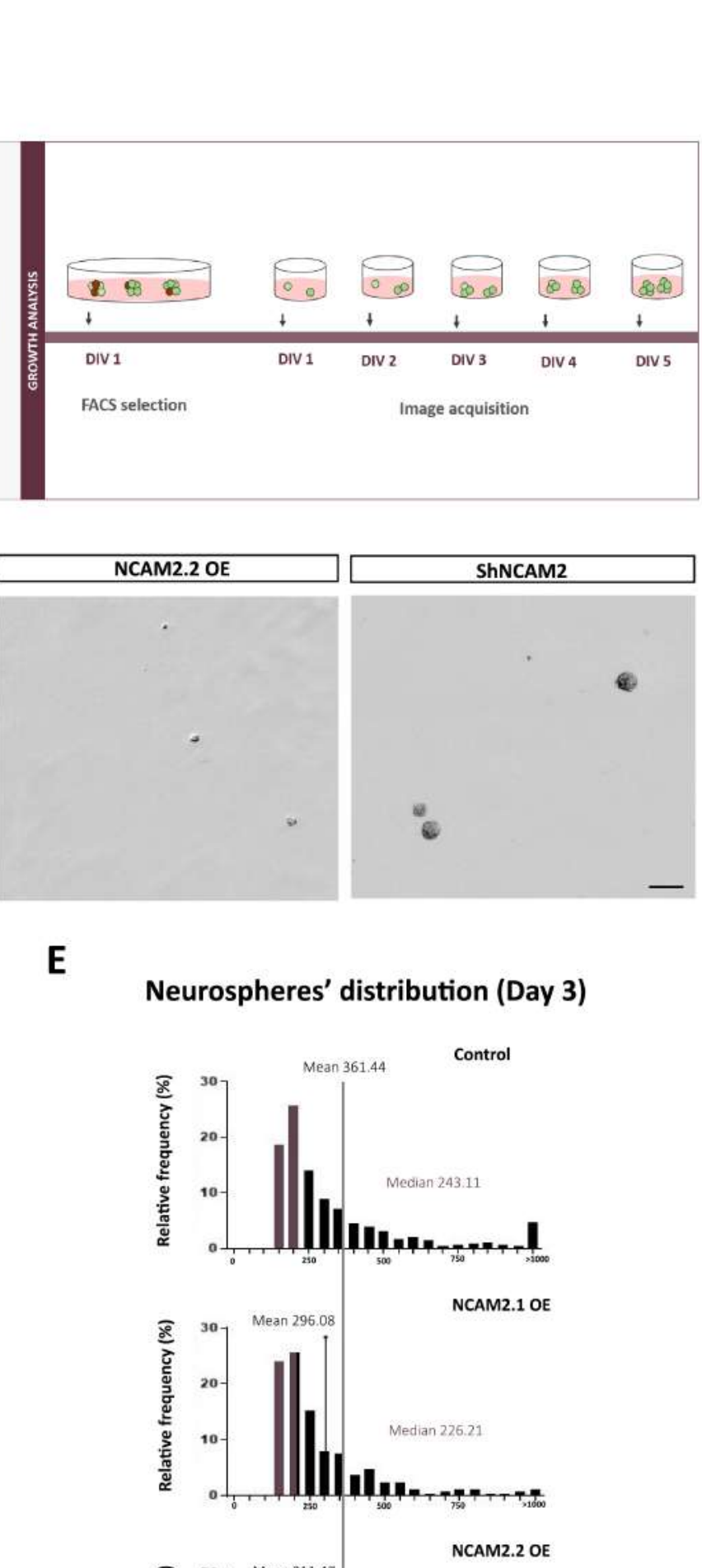

Neurospheres' distribution (Day 3)
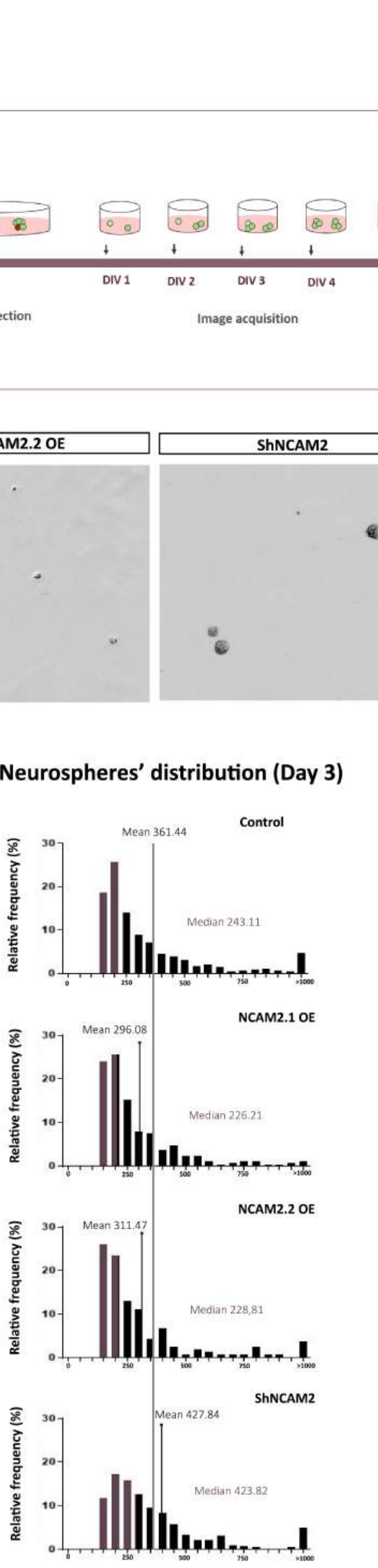

Area $(\mu \mathrm{m} 2)$

Figure 7 
Molecular

layer

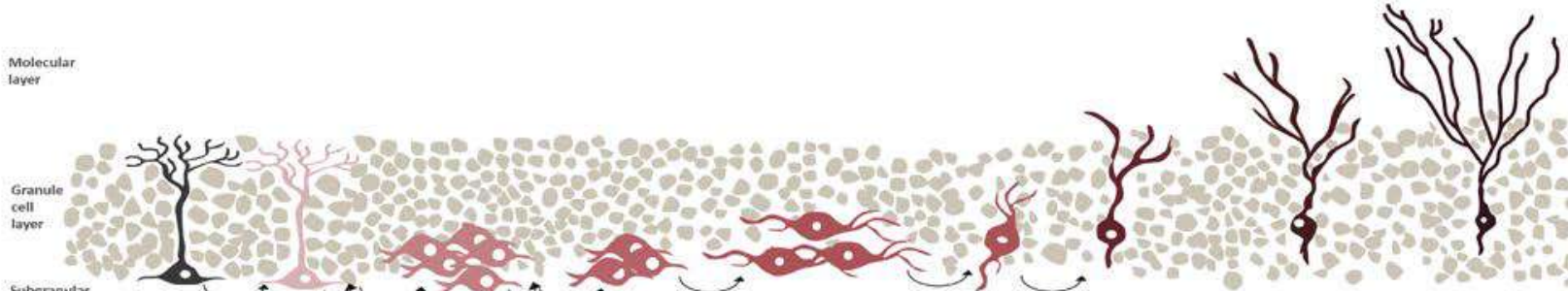

Subgranuta

Subgra
rone

$\underbrace{}_{\text {Type 1 cell }}$

INTERMEDIATE PROGENITORS

Type 3 cell

NEUROBLASTS

RGPs

INTE

(1)

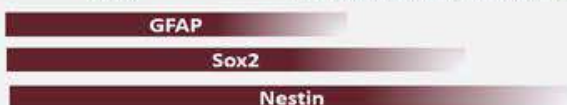

Thr2

NeuN

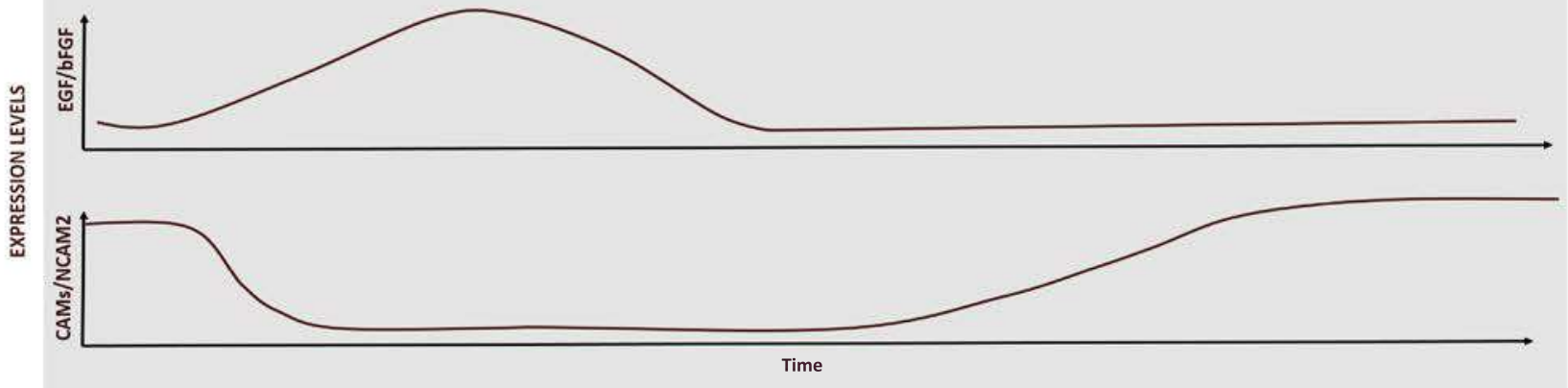

Figure 8 